\title{
Craig Interpolation with Clausal First-Order Tableaux
}

\author{
Christoph Wernhard
}

Version: March 21, 2021

\begin{abstract}
We develop foundations for computing Craig-Lyndon interpolants of two given formulas with first-order theorem provers that construct clausal tableaux. Provers that can be understood in this way include efficient machine-oriented systems based on calculi of two families: goal-oriented such as model elimination and the connection method, and bottom-up such as the hypertableau calculus. We present the first interpolation method for first-order proofs represented by closed tableaux that proceeds in two stages, similar to known interpolation methods for resolution proofs. The first stage is an induction on the tableau structure, which is sufficient to compute propositional interpolants. We show that this can linearly simulate different prominent propositional interpolation methods that operate by an induction on a resolution deduction tree. In the second stage, interpolant lifting, quantified variables that replace certain terms (constants and compound terms) by variables are introduced. We justify the correctness of interpolant lifting (for the case without built-in equality) abstractly on the basis of Herbrand's theorem and for a different characterization of the formulas to be lifted than in the literature. In addition, we discuss various subtle aspects that are relevant for the investigation and practical realization of first-order interpolation based on clausal tableaux.
\end{abstract}

Keywords Craig interpolation · first-order theorem proving · clausal tableaux · connection method $\cdot$ inductive interpolation $\cdot$ simulation between calculi $\cdot$ two-stage interpolation . interpolant lifting

\section{Introduction}

By Craig's interpolation theorem [23,24], ${ }^{1}$ for two first-order formulas $F$ and $G$ such that $F$ entails $G$ there exists a third first-order formula $H$ that is entailed by $F$, entails $G$ and is such that all predicate and function symbols and all free variables occurring in it occur in both $F$ and $G$. Such a Craig interpolant $H$ can be constructed from given formulas $F$ and $G$ with a calculus that allows to extract $H$ from a proof that $F$ entails $G$, or, equivalently, a proof that $F \rightarrow G$ is valid, or, again equivalently, a refutation of $F \wedge \neg G$. Automated construction

E-mail: info@christophwerhard.com

1 The development of Craig's interpolation theorem is described in [25]. 
of interpolants has many applications, in the area of computational logic most notably in symbolic model checking, initiated with [58], and in query reformulation [72,56, 64, 15, $76,19,7,37,9,8,77]$. The foundation for the latter application field is the observation that a reformulated query can be viewed as a definiens of a given query where only symbols from a given set, the target language of the reformulation, occur in the definiens. The existence of such definientia, that is, definability [75], or determinacy as it is called in the database context, can be expressed as validity and their synthesis as interpolant construction [24]. For example, a definiens $H$ of a unary predicate $\mathrm{p}$ within a first-order formula $F$ can be characterized by the following conditions:

1. $F$ entails $\forall x(\mathrm{p}(x) \leftrightarrow H)$.

2. $\mathrm{p}$ does not occur in $H$.

The variable $x$ is allowed there to occur free in $H$. We further assume that $x$ does not occur free in $F$ and let $F^{\prime}$ denote $F$ with $p$ replaced by a fresh symbol $\mathrm{p}^{\prime}$. Now the characterization of definiens by the two conditions given above can be equivalently expressed as

$H$ is a Craig interpolant of the two formulas $F \wedge \mathrm{p}(x)$ and $\neg\left(F^{\prime} \wedge \neg \mathrm{p}^{\prime}(x)\right)$.

A definiens $H$ exists if and only if it is valid that the first formula implies the second one.

There are many known ways to strengthen Craig's interpolation theorem by ensuring that if formulas $F$ and $G$ where $F$ entails $G$ satisfy certain syntactic restrictions, then there exists an interpolant $H$ that also satisfies certain syntactic restrictions. We consider here in particular that predicates occur in $H$ only with polarities with which they occur in both $F$ and $G$. (A predicate occurs with positive (negative) polarity in a formula if it occurs there in the scope of an even (odd) number of negation operators.) The respective strengthened interpolation theorem has been explicated by Lyndon [54], hence we call Craig interpolants that meet this additional restriction Craig-Lyndon interpolants.

\subsection{Craig Interpolation and Clausal Tableaux}

The construction of Craig interpolants of given first-order formulas has been elegantly specified in the framework of analytic tableaux by Smullyan [73,31]. Although this has been taken as foundation for applications of interpolation in query reformulation [76,9], it has been hardly used as a basis for the practical computation of first-order interpolants with automated reasoning systems, where so far mainly the interpolant extraction from resolution proofs, also with paramodulation and superposition, has been considered (see [13,14,45] for recent overviews and discussions). Some of these techniques only work on specially constrained proofs. In general they support equality handling with superposition and integrated theory reasoning, targeted at applications in verification.

Here we approach the computation of interpolants from another paradigm of automated reasoning, the framework of clausal tableaux (or clause tableau [38]) [47,49,48,52]. It has been established in the 1990s as a tool to develop and investigate methods for fully automated (in contrast to interactive) first-order theorem proving that operate not by manipulating clause sets (like resolution), but by creating a tree structure, the clausal tableau, that arranges copies of the input clauses. It is this explicit representation of the proof as a structure, the clausal tableau, that lends itself to inductive extraction of an interpolant.

Expectations are that, on the one hand, the elegance of Smullyan's interpolation method for analytic tableaux can be utilized and, on the other hand, a foundation for efficient practical implementations can be laid. 
The practically efficient theorem proving methods that can be viewed as operating by constructing a clausal tableau can be roughly divided into two major families. First, methods that are goal-sensitive, typically proceeding with the tableau construction "top-down", by "backward reasoning", starting with clauses from the theorem in contrast to the axioms. Aside of clausal tableaux in the literal sense, techniques to specify and investigate such methods include model elimination [53], the connection method [10,11], and the Prolog Technology Theorem Prover [74]. One of the leading first-order proving systems of the 1990s, SETHEO [51], followed that approach. The leanCoP system [66] along with its recent derivations [41, 42] as well as the CMProver component of PIE [26,81,83] are implementations in active duty today.

The second major family of methods constructs clausal tableaux "bottom-up", in a "forward reasoning" manner, by starting with positive axioms and deriving positive consequences. With the focus of their suitability to construct model representations, these methods have been called bottom-up model generation (BUMG) methods [4]. They include, for example, SATCHMO [18] and the hypertableau calculus [3], with implementations such as Hyper, formerly called E-KRHyper $[67,79,6]$. Hypertableau methods are also used in high-performance description logic reasoners [62]. It appears that the family of chase methods from the database field $[55,30]$, which recently got attention anew in knowledge representation (see, e.g., [34]), can also be understood as such a bottom-up tableau construction.

Depending on the method, the constructed clausal tableaux have particular structural characteristics, such as for top-down techniques typically the connectedness condition, which ensures that each inner node in the tableau has a child with complementary literal label. Bottom-up techniques are often applied in a way such that nodes labeled with a negative literal only appear as leaves. For a systematic overview of different variants of tableau structures and methods, including clausal tableaux realizing both major paradigms considered above see [38].

An essential feature that makes tableau methods particularly suitable for interpolation compared to methods based on resolution and paramodulation/superposition is that they generate only formulas which are instances of subformulas of the input. For clausal tableaux this means that only instances of input clauses are created. More generally, methods of the instance-based approach to theorem proving (see [39,5] for overviews) realize this principle. A resolution step, in contrast, recombines fragments of different clauses into a new clause. Nevertheless, clausal tableau methods might be complemented by preprocessors that perform operations which recombine clause fragments, for example predicate elimination, where all clauses with a given predicate are replaced by all non-tautological resolvents upon literals with that predicate if this results in a smaller clause set (see, e.g, [29,44,43]).

An essential feature that distinguishes clausal from analytic tableau is that with the clausal form only a particularly simple formula structuring has to be considered, in essence sets of clauses. Through preprocessing with conversion to prenex form and Skolemization, the handling of quantifications amounts for clausal tableau methods just to the handling of free variables, which are considered implicitly as universally quantified. There are different approaches for this: In free-variable tableaux variables are treated in a rigid manner, which means their scope is the whole tableau and they are considered as placeholders for arbitrary ground terms (see, e.g., [48, Sect. 4]). This is typical for the mentioned top-down methods. It may, however, complicate tableau construction, because the instantiation of a variable has effect on all its occurrences throughout the partially constructed tableau. Hence the mentioned bottom-up methods typically handle variables in other ways (see, e.g., [3] and [38, Sect. 3.2.4]). For our interpolant construction from given clausal tableaux it seems that the rigid view of variables is the most simple and straightforward. 
For propositional logic there are various known methods to extract an interpolant by an induction on a tree representation of a resolution proof (e.g., $[36,58])$, which are surveyed and presented in a common framework in [13]. As we will see, these interpolation methods for resolution proofs can be linearly simulated by the extraction of interpolants with an induction on a clausal tableau. These simulations are based on a known linear simulation of tree resolution, that is, resolution with the proof constrained to form a tree in contrast to an unrestricted dag, by clausal tableaux [49, Sect. 7.3], which is related to earlier results [69, Sect. 5.6]. Different interpolation methods for resolution proofs are reflected in slight variations of the involved translation of resolution deduction trees into clausal tableaux.

\subsection{The Two-Stage Approach to First-Order Interpolation}

The two-stage approach to interpolation proceeds by first computing with an induction on a proof structure an intermediate formula that is constrained in certain ways and can be lifted in a second stage to an interpolant. Lifting means there to replace terms by variables and prepending a quantifier prefix upon these. The two-stage approach seems to originate in [36], has been systematically investigated in [14], and is used also in $[1,45]$. The lifting step is justified in $[36,1]$ on the basis of a proof structure, with resolution/paramodulation and natural deduction, respectively, as underlying calculi. In [14] it is justified independently of a calculus, but applies only if the terms to be replaced by variables are constants.

It will be shown here that also the general case, where the terms to be replaced can be compound terms, leading to dependency constraints on the quantifications in the computed prefix, can be justified independently of a calculus and of proof structures on fundamental techniques of automated theorem proving, Skolemization and Herbrand's theorem.

The intermediate formula computed in the first stage is called in $[36,1,14]$ relational, weak and provisional interpolant, respectively, and is characterized with respect to the two interpolation inputs as satisfying the semantic constraints of a Craig interpolant, but the syntactic constraints only with respect to predicates (relational and weak interpolant) or not at all (provisional interpolant). Our view of lifting based on Herbrand's theorem allows to characterize the intermediate formula computed in the first stage as an actual Craig-Lyndon interpolant of two formulas that relate to the interpolation inputs. To justify interpolant lifting it is just required that these two formulas exist. They need not to be materialized at interpolant computation.

In the first stage of the two-stage method presented here a ground interpolant is extracted from a closed clausal ground tableau that represents a proof of the unsatisfiability of a set of first-order clauses. The construction process of the tableau is entirely independent from its use for interpolation, permitting to use existing provers without modification and utilizing their optimizations. Variables in the tableau returned from the prover must be rigid. Before the inductive interpolant extraction they are instantiated by ground terms, which is then a simple linear operation. Preprocessing has to be restricted, as for interpolation it is not sufficient to preserve just unsatisfiability of the input clauses.

\subsection{Summary of Contributions and Structure of the Paper}

The contributions of the paper can be summarized as:

1. An extension of the two-stage approach to Craig interpolation to first-order tableauxbased methods with rigid variables. 
2. A method for ground Craig-Lyndon interpolation by an induction on clausal tableaux obtained as proof representation from clausal first-order theorem provers. The method adapts a known interpolation method for analytic tableaux to clausal tableaux.

3. For propositional logic: Linear simulations of various known inductive interpolation methods for resolution proofs with the ground interpolation method based on clausal tableaux. Differences in the methods for resolution proofs correspond to variations in the translation of resolution deduction trees into clausal tableaux.

4. In the context of the two-stage approach to interpolation: A new justification for the second stage, interpolant lifting, that is based on Herbrand's theorem and independent of a calculus or proof structure. It provides a new characterization of the results of the formulas computed in the first stage as actual Craig-Lyndon interpolants of certain intermediate formulas for which a construction can be specified, but which need not to be actually constructed in the computation of first-order interpolants.

5. Discussion of various subtle aspects that are relevant for the investigation and practical realization of first-order interpolation, such as adequacy of the color-based terminology, interpolation-specific choices in the algorithms, and adaptations of preprocessing.

6. A basis for implementations of interpolation with efficient machine-oriented theorem provers for first-order logic that can be understood as constructing clausal tableaux. With methods and systems of two main families, goal-oriented "top-down" and forward reasoning "bottom-up", there is a wide range of potential applications.

The rest of the paper is structured as follows: After notation and basic terminology have been specified in Sect. 2, precise accounts of clausal tableau and related notions are given in Sect. 3. In Sect. 4 the inductive extraction of ground interpolants from closed clausal ground tableaux is specified and proven correct. The relationship to other propositional interpolation methods including the simulation of methods based on resolution proofs is shown in Sect. 5. We then turn to the lifting stage. In Sect. 6 additional notation is specified and the main theorem about interpolant lifting is stated in Sect. 7. It is applied in Sect. 8 to specify a two-stage interpolation procedure and proven in Sect. 9. Section 10 discusses related work, refinements of our method and some open issues for further research. Section 11 concludes the paper, summarizing its main contributions and indicating related additional research perspectives.

A work-in-progress poster of this research at an earlier stage was presented at the TABLEAUX 2017 conference.

\section{Notation and Basic Terminology}

Formulas. We basically consider first-order logic without equality. ${ }^{2}$ Atoms are of the form $p\left(t_{1}, \ldots, t_{n}\right)$, where $p$ is a predicate symbol (briefly predicate) with associated arity $n \geq 0$ and $t_{1}, \ldots, t_{n}$ are terms formed from function symbols (briefly functions) with associated arity $\geq 0$ and individual variables (briefly variables). Function symbols with arity 0 are also called individual constants (briefly constants). Unless especially noted, a formula is understood as a formula of first-order logic without equality, constructed from atoms, constant operators $T, \perp$, the unary operator $\neg$, binary operators $\wedge, \vee$ and quantifiers $\forall, \exists$ with their usual meaning. Further binary operators $\rightarrow, \leftrightarrow$ as well as $n$-ary versions of $\wedge$ and $\vee$ can be understood as meta-level shorthands. Also quantification upon a finite set of variables is used as shorthand for successive quantification upon each of its elements. The operators $\wedge$ and $\vee$

\footnotetext{
2 This does not preclude to represent equality as a predicate with axioms that express reflexivity, symmetry, transitivity and substitutivity.
} 
bind stronger than $\rightarrow$ and $\leftrightarrow$. The scope of $\neg$, the quantifiers, and the $n$-ary connectives is the immediate subformula to the right. Formulas in which no predicates with arity larger than zero and no quantifiers occur are called propositional.

Clausal Formulas. A literal is an atom or a negated atom. If $A$ is an atom, then the complement of $A$ is $\neg A$ and the complement of $\neg A$ is $A$. The complement of a literal $L$ is denoted by $\bar{L}$. A literal and its complement are said to be complementary. A clause is a (possibly empty) disjunction of literals. A clausal formula is a (possibly empty) conjunction of clauses, called the clauses in or the clauses of the formula. A clausal formula is a special case of a formula.

Variables, Vocabulary and Literals of a Formula. If $E$ is a term or a quantifier-free formula, then the set of variables occurring in $E$ is denoted by $\operatorname{Var}(E)$. If $F$ is a formula, then the set of variables that occur free in $F$ is denoted by $\mathcal{F}$ ree $\mathcal{V} \operatorname{Var}(F)$. A formula without free variables is called a sentence. A term or quantifier-free formula in which no variable occurs is called ground. A ground formula is thus a special case of a sentence. Symbols not present in the formulas and other items under discussion are called fresh.

If $E$ is a term or a formula, then the set of functions (including constants) occurring in $E$ is denoted by $\mathcal{F} u n(E)$. A subformula occurrence has in a given formula positive (negative) polarity, or is said to occur positively (negatively) in the formula, if it is in the scope of an even (odd) number of negations. If $F$ is a formula, then the set of predicates occurring in $F$ is denoted by $\operatorname{Pred}(F)$, and $\mathcal{P} r e d^{ \pm}(F)$ denotes the set of pairs $\langle p, p o l\rangle$, where $p$ is a predicate and $p o l \in\{+,-\}$, such that an atom with predicate $p$ occurs in $F$ with the polarity indicated by pol. For example, $\mathcal{P r e d}^{ \pm}(\mathrm{p})=\{\langle\mathrm{p},+\rangle\}$ and $\mathcal{P r e d}^{ \pm}(\mathrm{p} \vee \neg \mathrm{p})=\{\langle\mathrm{p},+\rangle,\langle\mathrm{p},-\rangle\}$. We define $\mathcal{V o c}(F) \stackrel{\text { def }}{=} \operatorname{Pred}(F) \cup \mathcal{F} u n(F)$ and $\mathcal{V o c}^{ \pm}(F) \stackrel{\text { def }}{=} \mathcal{P r e d}^{ \pm}(F) \cup \mathcal{F} u n(F)$.

If $F$ is a formula, then the set of pairs $\langle A, p o l\rangle$, where $A$ is an atom and pol $\in\{+,-\}$ such that $A$ occurs in $F$ with the polarity indicated by pol is denoted by Literals $(F)$. Observe that since $T$ and $\perp$ are not considered as atomic formulas but as constant logical operators it holds that $\mathcal{L}$ iterals $(T)=\mathcal{L}$ iterals $(\perp)=\emptyset$.

The notation $\operatorname{Var}(E)$ and $\mathcal{F} u n(E)$ is also used with sets $E$ of terms or formulas, where it stands for the union of values of the respective function applied to each member of $E$.

Semantic Relationships. We write $F \models G$ for $F$ entails $G$, and $F \equiv G$ for $F$ is equivalent to $G$, that is, $F \models G$ and $G \models F$. On occasion we write a sequence of statements with these operators where the right and left, respectively, arguments of subsequent statements are identical in a chained way, such as, for example, $F \mid G \models H$ for $F \mid=G$ and $G \models H$.

\section{Clausal First-Order Tableaux}

The following definition makes the variant of clausal tableaux that we use as basis for interpolation precise. It is targeted at modeling tableau structures produced by efficient fully automated first-order proving systems based on different calculi.

\section{Definition 1 (Clausal Tableau and Related Notions)}

(i) Let $F$ be a clausal formula. A clausal tableau (briefly tableau) for $F$ is a finite ordered tree whose nodes $N$ with exception of the root are labeled with a literal, denoted by $\operatorname{lit}(N)$, such that the following condition is met: For each node $N$ of the tableau the disjunction of the labels of all its children in their left-to-right order, denoted by clause $(N)$, is an instance 
of a clause in $F$. A value of clause $(N)$ for a node $N$ in a tableau is called a clause of the tableau.

(ii) A branch of a tableau is called closed if and only if it contains nodes with complementary literals. A node $N$ is called closed if and only if all branches through $N$ are closed. A tableau is called closed if and only if its root is closed.

(iii) A node of a tableau is called closing if and only if it has an ancestor with complementary literal. With a closing node $N$, a particular such ancestor is associated as target of $N$, written $\operatorname{tgt}(N)$. A tableau is called leaf-closed if and only if all of its leaves are closing.

(iv) A tableau is called ground if and only if for all its nodes $N$ it holds that $\operatorname{lit}(N)$ is ground.

The most immediate relationship of clausal tableaux to the semantics of clausal formulas is that the universal closure of a clausal formula is unsatisfiable if and only if there exists a closed clausal tableaux for the clausal formula. Knowing that there are sound and complete calculi that operate by constructing a closed clausal tableau for an unsatisfiable clausal formula, and taking into account Herbrand's theorem we can state the following proposition.

Proposition 2 (Unsatisfiability and Computation of Closed Clausal Tableaux) There is an effective method that computes from a clausal formula $F$ a closed clausal tableau for $F$ if and only if $\forall x_{1} \ldots \forall x_{n} F$, where $\left\{x_{1}, \ldots, x_{n}\right\}=\mathcal{V a r}(F)$, is unsatisfiable. Moreover, this also holds if terms in the literal labels of tableau nodes are constrained to ground terms formed from functions occurring in $F$ and, in case there is no constant occurring in $F$, an additional fresh constant.

Our objective is here interpolant construction on the basis of clausal tableaux produced by fully automated systems. This has effect on some aspects of our formal notion of clausal tableau: All occurrences of variables in the literal labels of a tableau according to Definition 1.i are free and the scope of these variables spans all literal labels of the whole tableau. In more technical terms, this means that the tableaux are free-variable tableaux (see [48, p. 158ff]) with rigid variables (see [38, p. 114]).

The closing property of nodes, the target function, and the leaf-closed property of tableaux are introduced to facilitate the definition of interpolants by induction on the tableau structure, bottom-up, with leaves considered in the base cases. It is easy to see that any closing node is also a closed node and that any leaf-closed tableau is also a closed tableau. Any closed tableau for a clausal formula $F$ can be easily converted to a leaf-closed tableau for $F$ by removing all edges that originate in nodes that are closing. Practical proving methods typically construct a closed tableau that is already leaf-closed, since for the construction of a closed tableau it is pointless to attach children to a closing, and hence closed, node.

\section{Ground Interpolation with Clausal Tableaux}

Craig's interpolation theorem along with Lyndon's observation ensures the existence of Craig-Lyndon interpolants, defined as follows.

Definition 3 (Craig-Lyndon Interpolant) Let $F, G$ be formulas such that $F=G$. A CraigLyndon interpolant of $F$ and $G$ is a formula $H$ such that

1. $F \models H \models G$.

2. $\mathcal{V} o c^{ \pm}(H) \subseteq \mathcal{V} o c^{ \pm}(F) \cap \mathcal{V} o c^{ \pm}(G)$.

3. Free $\operatorname{Var}(H) \subseteq \mathcal{F}$ ree $\operatorname{Var}(F) \cap \mathcal{F}$ ree $\operatorname{Var}(G)$. 
If condition (2) is replaced by the weaker condition $\mathcal{V o c}(H) \subseteq \mathcal{V} o c(F) \cap \mathcal{V o c}(G)$, that is, the polarity of predicate occurrences in $H$ is not taken into account, then we call $H$ a Craig interpolant. Many automated reasoning techniques, including the clausal tableau methods considered here, validate an entailment $F \mid=G$ by showing the unsatisfiability of $F \wedge \neg G$. In the literature on interpolation in verification this is reflected in the notion of reverse interpolant.

Definition 4 (Reverse Craig-Lyndon Interpolant) Let $F, G$ be formulas such that $F \wedge G \models$ $\perp$. A reverse Craig-Lyndon interpolant of $F$ and $G$ is then defined as a Craig-Lyndon interpolant of $F$ and $\neg G$.

Our interpolant construction is based on a variant of clausal tableaux where nodes have an additional side label that is shared by siblings and indicates whether the tableau clause is an instance of an input clause derived from the formula $F$ or the formula $G$ of the statement $F \wedge G \models \perp$ underlying the reverse interpolant.

\section{Definition 5 (Two-Sided Clausal Tableau and Related Notions)}

(i) Let $F, G$ be clausal formulas. A two-sided clausal tableau for $F$ and $G$ (or briefly tableau for the two formulas) is a clausal tableau for $F \wedge G$ whose nodes $N$ with exception of the root are labeled additionally with a side side $(N) \in\{\mathrm{F}, \mathrm{G}\}$, such that the following conditions are met by all nodes $N, N^{\prime}$ of the tableau:

1. If $N$ and $N^{\prime}$ are siblings, then $\operatorname{side}(N)=\operatorname{side}\left(N^{\prime}\right)$.

2. If $N$ has a child $N^{\prime}$ with side $\left(N^{\prime}\right)=\mathrm{F}$, then clause $(N)$ is an instance of a clause in $F$. If $N$ has a child $N^{\prime}$ with side $\left(N^{\prime}\right)=\mathrm{G}$, then clause $(N)$ is an instance of a clause in $G$.

We also refer to the side of the children of a node $N$ as side of clause $(N)$.

(ii) For side $\in\{\mathrm{F}, \mathrm{G}\}$ and all nodes $N$ of a two-sided clausal tableau define

$$
\operatorname{path}_{\text {side }}(N) \stackrel{\text { def }}{=} \bigwedge_{N^{\prime} \in \text { Path and side }\left(N^{\prime}\right)=\text { side }} \operatorname{lit}\left(N^{\prime}\right),
$$

where Path is the union of the set of the ancestors of $N$ and $\{N\}$.

For examples of two-sided tableaux see Figure 1 and 2 in Example 9 below (ignore the node annotations in brackets). For examples of path consider Figure 2. Let $N$ be the rightmost node labeled with e there (above the rightmost leaf labeled with $\neg \mathrm{e}$ ). $\operatorname{Then} \operatorname{path}_{\mathrm{F}}(N)=\neg \mathrm{a} \wedge \mathrm{e}$ and $\operatorname{path}_{\mathrm{G}}(N)=\neg \mathrm{d} \wedge \neg \mathrm{c}$.

The literature on interpolation targeted at applications in verification uses a conceptualization with varying terminology (see [13] for an overview), which is related to the side labeling but would not exactly match our needs. The main conceptual difference is that our labeling applies to occurrences of symbols, terms and formulas, identified by tableau nodes, in contrast to symbols, terms and formulas themselves. Analogously, first-order interpolation methods based on resolution with paramodulation are specified with labelings that refer to occurrences in proof trees, is from [36] and label [14]. ${ }^{3}$

3 Symbols that appear only in $F$ or $G$ are called $F$-colored and $G$-colored, respectively, or colored in general (also local has been used synonymously to colored), while the other symbols are called transparent (or grey). It appears that the association with occurrences is in particular necessary to take account of predicate polarity required by Craig-Lyndon (in contrast to just $\mathrm{Craig}$ ) interpolants. Another reason for the occurrencebased labeling is that the possibility that some clause $C$ is an instance of a clause in $F$ as well as an instance of a clause in $G$ should be retained. An occurrence of $C$ then can be associated with either side label. A 
Definition 6 (Interpolant Extraction from a Clausal Tableau) Let $N$ be a node of a leafclosed two-sided clausal tableau. The value of ipol $(N)$ is a quantifier-free formula, defined inductively as follows:

i. If $N$ is a leaf, then the value of $\operatorname{ipol}(N)$ is determined by the values of $\operatorname{side}(N)$ and side $(\operatorname{tgt}(N))$ as specified in the following table:

\begin{tabular}{ccc}
$\operatorname{side}(N)$ & side $(\operatorname{tgt}(N))$ & $\operatorname{ipol}(N)$ \\
\hline $\mathrm{F}$ & $\mathrm{F}$ & $\perp$ \\
$\mathrm{F}$ & $\mathrm{G}$ & $\operatorname{lit}(N)$ \\
$\mathrm{G}$ & $\mathrm{F}$ & $\frac{\operatorname{lit}(N)}{\mathrm{G}}$ \\
$\mathrm{G}$ & $\mathrm{G}$ & $\mathrm{T}$
\end{tabular}

ii. If $N$ is an inner node with children $N_{1}, \ldots, N_{n}$ where $n \geq 1$, then the value of $\operatorname{ipol}(N)$ is composed from the values of ipol for the children, disjunctively or conjunctively, depending on the side label of the children (which is the same for all of them), as specified in the following table:

\begin{tabular}{cc}
$\operatorname{side}\left(N_{1}\right)$ & $\operatorname{ipol}(N)$ \\
\hline $\mathrm{F}$ & $\bigvee_{i=1}^{n} \operatorname{ipol}\left(N_{i}\right)$ \\
$\mathrm{G}$ & $\bigwedge_{i=1}^{n} \operatorname{ipol}\left(N_{i}\right)$
\end{tabular}

The following lemma gives semantic and syntactic properties of the formula returned by applying ipol to the root of a leaf-closed ground tableau for ground formulas. ${ }^{4}$ These properties imply the conditions required from a Craig-Lyndon interpolant as specified in Definition 3.

Lemma 7 (Correctness of Interpolant Extraction from Clausal Ground Tableaux) Let $F, G$ be clausal ground formulas and let $N_{0}$ be the root of a leaf-closed two-sided clausal ground tableau for $F$ and $G$. Then

1. $F \models \operatorname{ipol}\left(N_{0}\right) \models \neg G$.

2. L Literals $\left(\operatorname{ipol}\left(N_{0}\right)\right) \subseteq \mathcal{L}$ iterals $(F) \cap \mathcal{L}$ iterals $(\neg G)$.

Proof We show that the following invariant of ipol holds for all nodes $N$ of the tableau, including the root, which immediately implies the proposition:

perhaps confusing aspect of the color-based terminology is that the term colored on the one hand indicates for a symbol that it appears only in one of $F$ or $G$, a property called isolated in [24], while, on the other hand, the colored property for compound structures, that is, terms and formulas, permits occurrences of transparent symbols. We apply the color-based terminology in Sect. 5 and discuss it further in the context of first-order interpolation at the end of Sect. 7. Limitations of the color-based approaches are also discussed in [14, Sect. 3].

4 The ipol function is also defined on non-ground tableaux, as it is independent of occurrences of variables. However, the association with properties relevant for interpolation is simplified if we assume a ground tableau, which is without loss of generality: A calculus may construct as proof a closed free-variable tableau with occurrences of free rigid variables. Any ground instantiation of these variables yields a ground tableau that provides a proof of the same formula. In particular, an instantiation of each variable by a dedicated constant. For the considered properties, tableaux with variables can be represented by ground tableaux with such constants. The restriction that the tableau is for ground formulas provides another simplification that, however, does not restrict the applicability of the lemma in a first-order context: A ground tableau that provides a proof of a clausal first-order formula also provides a proof of a clausal ground formula, the conjunction of the tableau clauses. In the setting of two-sided tableaux this can be stated more precisely as: A leaf-closed two-sided ground tableau for two clausal first-order formulas is also a leaf-closed two-sided ground tableau for two clausal ground formulas, the conjunction of the tableau clauses with side F and the conjunction of the tableau clauses with side $\mathrm{G}$. 
(a) $F \wedge \operatorname{path}_{F}(N) \vDash \operatorname{ipol}(N) \mid=\neg G \vee \neg \operatorname{path}_{\mathrm{G}}(N)$.

(b) L Literals $(\operatorname{ipol}(N)) \subseteq \mathcal{L}$ iterals $\left(F \wedge \operatorname{path}_{\mathrm{F}}(N)\right) \cap \mathcal{L}$ iterals $\left(\neg G \vee \neg \operatorname{path}_{\mathrm{G}}(N)\right)$.

This is proven by induction on the tableau structure, proceeding from leaves upwards. We prove the base case, where $N$ is a leaf, by showing (a) and (b) for all possible values of side $(N)$ and side $(\operatorname{tgt}(N))$ :

- $\operatorname{Case} \operatorname{side}(N)=\mathrm{F}$ :

- Case side $(\operatorname{tgt}(N))=\mathrm{F}$ : Immediate since then $\operatorname{path}_{\mathrm{F}}(N) \models \perp=\operatorname{ipol}(N)$.

- Case side $(\operatorname{tgt}(N))=\mathrm{G}$ : Then $\operatorname{ipol}(N)=\operatorname{lit}(N)$. Properties (a) and (b) follow because $\operatorname{lit}(N)$ is a conjunct in $\operatorname{path}_{\mathrm{F}}(N)$ and $\operatorname{lit}(N)$ is a conjunct in $\operatorname{path}_{\mathrm{G}}(N)$.

- $\operatorname{Case} \operatorname{side}(N)=\mathrm{G}$ :

- Case side $(\operatorname{tgt}(N))=\mathrm{F}$ : Then $\operatorname{ipol}(N)=\overline{\operatorname{lit}(N)}$. Properties (a) and (b) follow because lit $(N)$ is a conjunct in $\operatorname{path}_{\mathrm{F}}(N)$ and $\operatorname{lit}(N)$ is a conjunct in path $\operatorname{Ga}_{\mathrm{G}}(N)$.

- Case side $(\operatorname{tgt}(N))=\mathrm{G}$ : Immediate since then $\operatorname{ipol}(N)=\mathrm{T} \models \neg \operatorname{path}_{\mathrm{G}}(N)$.

To show the induction step, assume that $N$ is an inner node with children $N_{1}, \ldots, N_{n}$. Consider the case where the side of the children is $F$. The induction step for the case where the side of the children is $\mathrm{G}$ can be shown analogously. By the induction hypothesis we can assume that for all $i \in\{1, \ldots, n\}$ it holds that

$$
F \wedge \operatorname{path}_{\mathrm{F}}\left(N_{i}\right) \models \operatorname{ipol}\left(N_{i}\right) \models \neg G \vee \neg \operatorname{path}_{\mathrm{G}}\left(N_{i}\right),
$$

which, since $\operatorname{side}\left(N_{i}\right)=\mathrm{F}$, is equivalent to

$$
F \wedge \operatorname{path}_{\mathrm{F}}(N) \wedge \operatorname{lit}\left(N_{i}\right) \vDash \operatorname{ipol}\left(N_{i}\right) \vDash \neg G \vee \neg \operatorname{path}_{\mathrm{G}}(N) .
$$

This implies

$$
F \wedge \operatorname{path}_{\mathrm{F}}(N) \wedge \bigvee_{i=1}^{n} \operatorname{lit}\left(N_{i}\right) \models \bigvee_{i=1}^{n} \operatorname{ipol}\left(N_{i}\right) \vDash \neg G \vee \neg \operatorname{path}_{\mathrm{G}}(N) .
$$

Since ipol $(N)=\bigvee_{i=1}^{n}$ ipol $\left(N_{i}\right)$, according to the definition of ipol for nodes $N$ whose children have side $F$, it follows that

$$
F \wedge \operatorname{path}_{\mathrm{F}}(N) \wedge \bigvee_{i=1}^{n} \operatorname{lit}\left(N_{i}\right) \models \operatorname{ipol}(N) \models \neg G \vee \neg \operatorname{path}_{\mathrm{G}}(N) .
$$

Because $\bigvee_{i=1}^{n} \operatorname{lit}\left(N_{i}\right)=\operatorname{clause}(N)$ is by construction of the tableau a clause in $F$ and thus entailed by $F$ the semantic requirement (a) of the induction conclusion follows:

$$
F \wedge \operatorname{path}_{\mathrm{F}}(N) \vDash \operatorname{ipol}(N) \mid \neg G \vee \neg \operatorname{path}_{\mathrm{G}}(N) .
$$

The syntactic requirement (b) follows from the induction hypothesis and because in general for all nodes $N$ of a two-sided clausal ground tableau for clausal ground formulas $F$ and $G$ it holds that all literals in $\operatorname{path}_{\mathrm{F}}(N)$ occur in some clause of $F$ and all literals in $\operatorname{path}_{\mathrm{G}}(N)$ occur in some clause of $G$.

Lemma 7 immediately yields a construction method for Craig-Lyndon interpolants of propositional and, more general, first-order formulas that are ground (and without equality, except if represented as predicate, see Sect. 10.4). We call the procedure CTI, suggesting Clausal Tableau Interpolation. In Sect. 8 below it will be generalized to first-order sentences in full. 
Procedure 8 (The CTI Method for Craig-Lyndon Interpolation on Ground Formulas) InPuT: Ground formulas $F$ and $G$ such that $F \mid=G$.

METHOD:

1. Clausification. Convert $F$ and $\neg G$ to equivalent clausal ground formulas $F^{\prime}$ and $G^{\prime}$, respectively, such that $\mathcal{V} o c^{ \pm}\left(F^{\prime}\right) \subseteq \mathcal{V} o c^{ \pm}(F)$ and $\mathcal{V}_{o c}^{ \pm}\left(G^{\prime}\right) \subseteq \mathcal{V} \operatorname{Voc}^{ \pm}(\neg G)$.

2. Tableau computation. Compute a closed clausal ground tableau for $F^{\prime} \wedge G^{\prime}$. If the tableau is not already leaf-closed, convert it to leaf-closed form by removing all edges that originate in closing nodes.

3. Side assignment. Convert the ground tableau to a two-sided tableau for $F^{\prime}$ and $G^{\prime}$ by attaching appropriate side labels to all nodes except the root. This is always possible because every clause of the tableau is in $F^{\prime}$ or in $G^{\prime}$. (As $F$ and $G$ can be arbitrary ground formulas it is possible that a clause of the tableau appears in both $F^{\prime}$ and in $G^{\prime}$. For siblings corresponding to such a clause, the side labels can be either set all to $F$ or all to G. As shown in Example 10 below, this choice can have an effect on the computed interpolant. The issue is discussed further in Sect. 10.2.)

4. Interpolant extraction. Let $H$ be the value of ipol $\left(N_{0}\right)$, where $N_{0}$ is the root of the tableau.

Output: Return $H$. The output is a ground formula that is a Craig-Lyndon interpolant of the input formulas.

That the procedure is correct, which means that it outputs indeed a Craig-Lyndon interpolant of the input formulas, follows from Lemma 7 and Definition 3. The procedure computes an interpolant on the basis of any leaf-closed two-sided clausal ground tableaux for the clausified inputs $F^{\prime} \wedge G^{\prime}$, which follows from the definition of ipol and Lemma 7. (For interpolation systems that operate on resolution proofs, the analog to this property is termed complete relative to an inference system in [13, Def. 10].) Hence, if the invoked method for tableau computation is complete, that is, it outputs a closed clausal tableau for all unsatisfiable inputs, then the overall interpolation procedure is also complete, that is, it outputs a Craig-Lyndon interpolant of its inputs $F$ and $G$ whenever $F \models G$.

The size of the resulting formula is linear in the size of the tableau, or more precisely in the number of its leaves whose target has the opposite side label (assuming that truth value simplification ${ }^{5}$ is integrated into the computation of ipol). The size of the tableau itself is not polynomially bounded in the size of the clausal formulas underlying the tableau construction. ${ }^{6}$

The potential blow up in the transformation to clausal form can be avoided by replacing this step with the transformation to a structure preserving (also known as definitional) normal form, which needs to be applied for interpolation in a way such that the sets of auxiliary symbols introduced for transforming $F$ and $\neg G$ are disjoint. This is discussed further in Sect. 10.3.

Example 9 (Propositional Interpolation with Clausal Tableaux) To facilitate comparison with other methods for computing reverse interpolants for propositional clausal formulas we consider interpolation inputs from [13, Example 2]: Let $F=F^{\prime}=(a \vee e) \wedge(\neg a \vee b) \wedge(\neg a \vee c)$ and let $\neg G=G^{\prime}=(\neg \mathrm{b} \vee \neg \mathrm{c} \vee \mathrm{d}) \wedge \neg \mathrm{d} \wedge \neg \mathrm{e}$. As there are usually quite different leaf-closed

\footnotetext{
5 Exhaustively rewriting with $F \wedge \perp \equiv \perp, F \vee T \equiv \mathrm{T}, F \wedge \mathrm{T} \equiv F, F \vee \perp \equiv F$, modulo commutativity.

6 An argument for this is that even clausal tableaux with atomic cut, which can polynomially simulate tree resolution, can not polynomially simulate unrestricted resolution where proofs may have the form of dags [49].
} 
clausal tableaux for a given clausal formulas, we illustrate the assignment of values of ipol for two different such tableaux, both for $F^{\prime}$ and $G^{\prime}$. Figure 1 represents a typical result of a bottom-up calculus and Figure 2 of a top-down calculus, as further discussed below. In these figures, nodes with exception of the root are represented by their literal label. Nodes with side $\mathrm{G}$ are indicated by gray background. The remaining nodes have side F. For each node the value of ipol is annotated in brackets, where values are shown exactly as specified in Definition 6 except that truth-value simplification (see footnote 5) is applied.

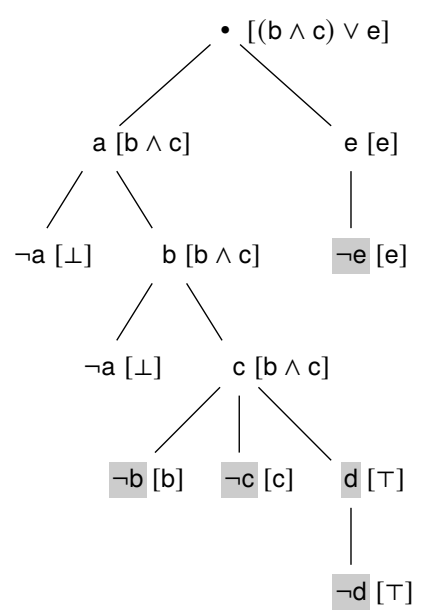

Fig. 1: Interpolation with a bottom-up constructed tableau.

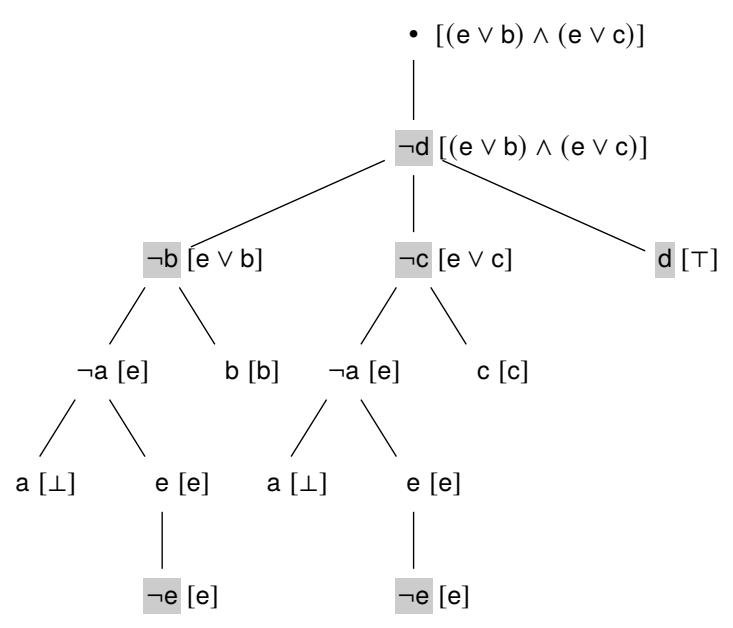

Fig. 2: Interpolation with a top-down constructed tableau.

The way in which a tableau was constructed step-by-step by a calculus is irrelevant for interpolant extraction. Nevertheless, the tableaux in Figure 1 and 2 suggest two different specific construction possibilities which we will sketch now. Both might have been constructed with calculi that start with the root and repeatedly extend an open (i.e., not closed) branch by attaching a clause. In the tableau of Figure 1 a clause is only attached if the complements of all its negative literals appear on the branch, with the effect that the tableau never has an open branch that ends with a negative literal. The clause a $\vee$ e, for example, has no negative literal and can thus be attached directly below the root. The clause $\neg \mathrm{b} \vee \neg \mathrm{c} \vee d$, for example, is attached to a branch in which $b$ and $c$ appear. Branches ending in $\neg b$ and $\neg c$ are created but immediately closed. Although presented as a tableau construction that starts with the root node, calculi that proceed in the indicated way may be understood as bottom-up methods, as they start from disjunctions of facts (positive clauses) and the extension of a branch by a clause is like the forward application of a rule: The antecedent is on the branch in the form of the complements of the negative clause literals and the consequent corresponds to its positive literals, each forming the end of a new open branch. Extension with a goal (negative clause) closes an open branch without introducing a new one.

The tableau of Figure 2 might have been constructed with a top-down method. Some clause is chosen as start clause and attached below the root, $\neg d$ in the example. Open branches are only extended with clauses such that the connection condition is preserved, that is, the complement of the last literal on the branch appears in the clause. The complement of the last literal on an open branch is viewed as an open subgoal. Extending the branch by attaching a clause replaces it with other subgoals or, if all literals of the clause have complements on the 
branch, solves it. Top-down tableau construction typically may lead to the necessity to solve the same subgoal more than once, as can be seen in the example with the replicated subtree at the two nodes labeled with $\neg$ a.

Returning to the question of interpolant extraction from a given clausal tableaux, the following example shows a case where the obtained interpolant depends on a choice of the side assignment step of Procedure 8.

Example 10 (Alternate Possible Side Assignments) Let $F=\mathrm{a} \wedge \mathrm{b} \wedge(\mathrm{b} \rightarrow \mathrm{c})$ and $G=$ $\mathrm{c} \vee \neg(\mathrm{b} \rightarrow \mathrm{c}) \vee \mathrm{d}$. Figures 3 and 4 each show a leaf-closed two-sided clausal tableau for the corresponding clausal formulas $F^{\prime}=a \wedge b \wedge(\neg b \vee c)$ and $G^{\prime}=\neg c \wedge(\neg b \vee c) \wedge \neg d$ according to Procedure 8. The tableaux are identical, except that for the clause $\neg \mathrm{b} \vee \mathrm{c}$, which is in $F^{\prime}$ and also in $G^{\prime}$, the side assigned to its occurrence in the tableau is different. The symbolism for indicating sides and values of ipol is as in Example 9. The overall interpolants of $F$ and $G$ returned by the procedure are $c$ and $b$, respectively.

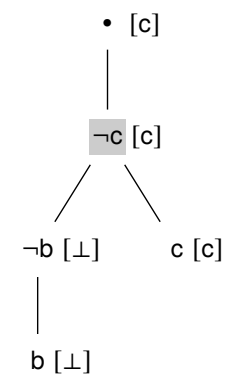

Fig. 3: Interpolation with $F$ as side of $\neg b \vee c$.

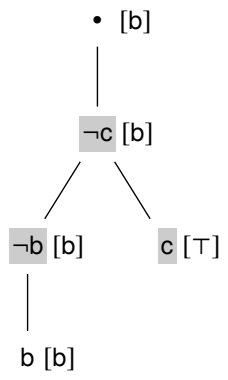

Fig. 4: Interpolation with $G$ as side of $\neg b \vee c$.

\section{Related Methods for Propositional Interpolation}

The extraction of interpolants from clausal tableaux with the ipol function emerged from a straightforward adaptation of the propositional part of Smullyan's interpolation method for analytic tableaux described in [73, Chap. XV] and [31, Chap. 8.12]. Differently from these works, the adaptation specifies the interpolant extraction not in terms of tableau manipulation rules that deconstruct the tableau bottom-up, but inductively, as a function that maps each node to a formula. It turned out that the clausal adaptation of Smullyan's method has some striking similarities to a family of interpolation methods for resolution proofs including, for example, the algorithms from [36] and [58], surveyed in a common framework in [13]. Like the tableau-based method, these interpolation methods for resolution proofs are specified by an induction on a proof structure, which led to the approach being called inductive [13].

The role of the clausal tableau for an unsatisfiable clausal formula $F$ is taken by a deduction tree [20] for $F$, that is, a finite ordered tree that represents a resolution proof. Its nodes are labeled with clauses such that each leaf is labeled by a clause in $F$, each inner node has exactly two children and is labeled by a binary resolvent of the clauses of its children, and the root is labeled with the empty clause $\square$. We assume that the involved clauses do not contain duplicate literals by expecting this from the clauses in $F$ and by assuming that the used variant of binary resolution incorporates merging of duplicate literals.

The invariants that justify the involved inductions for tableaux and for deduction trees are actually quite similar. For clausal tableaux, they have been stated above as (a) and (b) in the 
proof of Lemma 7. For deduction trees they can be expressed as follows: Let pi be a function that, analogously to ipol, maps each node in the deduction tree to a formula, the partial interpolant [13] associated with the node, let clause $(M)$ denote the clause label of deduction tree node $M$, let $\left.C\right|_{\mathrm{F}}$ denote the clause $C$ with all literals deleted whose predicate does not occur in $F$, and, analogously, let $\left.C\right|_{\mathrm{G}}$ denote $C$ with all literals deleted whose predicate does not occur in $G$. Based on [36, Proof of Theorem 2] and [13, Proposition 9], the invariants expected to hold for all nodes $M$ of the deduction tree can then be stated as

(a') $F \wedge \neg$ clause $\left.(M)\right|_{\mathrm{F}} \vDash \mathrm{pi}(M) \models \neg G \vee$ clause $\left.(M)\right|_{\mathrm{G}}$.

$\left(\mathrm{b}^{\prime}\right) \operatorname{Pred}(\operatorname{pi}(N)) \subseteq \operatorname{Pred}(F) \cap \operatorname{Pred}(G)$.

If $M_{0}$ is the root of the deduction tree, labeled with the empty clause $\square$, then clause $\left.\left(M_{0}\right)\right|_{\mathrm{F}}=$ clause $\left.\left(M_{0}\right)\right|_{\mathrm{G}}=\perp$ and the invariants $\left(\mathrm{a}^{\prime}\right)$ and $\left(\mathrm{b}^{\prime}\right)$ imply that $\mathrm{pi}\left(M_{0}\right)$ is an interpolant of $F$ and $\neg G$. There is a parallelism between the roles of the clause projections clause $\left.(M)\right|_{\mathrm{F}}$ and clause $\left.(M)\right|_{G}$ in invariant $\left(\mathrm{a}^{\prime}\right)$ for deduction trees and the path projections $\operatorname{path}_{\mathrm{F}}(N)$ and path $_{\mathrm{G}}(N)$ in invariant (a) of Lemma 7 for clausal tableaux.

A profound account of these similarities is an issue for future research. As a step in this direction, we show that various inductive propositional interpolation methods for resolution proofs can be linearly simulated with our clausal tableau method. We show this in detail for Huang's algorithm [36], an early method, whose fundamental role has been observed only later with $[12,13,14]$. The simulations suggest a new way to systematize interpolation methods for resolution proofs and, as they involve just linear-time conversions from treeshaped resolution proofs, may be useful for practical implementation.

The basis for these simulations are clausal tableaux with atomic cuts. The atomic cut rule [50] permits to extend a clausal tableau under construction by attaching to a node two successors, labeled with $A$ and $\neg A$, respectively, where $A$ is an arbitrary atom. Equivalently, this can be considered as attaching siblings whose clause is the tautology $\neg A \vee A$, also if that tautology is not an input clause. The atomic cut rule is of interest for theorem proving as it permits substantially shorter proofs. Simulation by clausal tableaux with atomic cut is a central tool in the analysis of advanced construction techniques for clausal tableaux as well as investigations of relationships of clausal tableau methods to other calculi $[49,50]$. Of particular relevance for our simulations of interpolation methods is that, for propositional logic, clausal tableaux with atomic cut can linearly simulate tree resolution, that is, resolution where the proof graph forms a tree in contrast to an unrestricted dag, which has been shown via the semantic tree method as an intermediate method in [49, Prop 7.33 and 7.40]. ${ }^{7}$ The simulation is achieved with an encoding of a deduction tree as a closed clausal tableau with atomic cuts, that is, a tableau that permits interspersed "cuts", tautological clauses of the form $\neg A \vee A$ that are not necessarily in the input formula. Moreover, the clausal tableaux that encode resolution deduction trees are in cut normal form [49], that is, all their clauses except those of nodes whose children are all leaves are "cuts" of the form $\neg A \vee A$.

This encoding can be modified, preserving linearity and the cut normal form, to produce a leaf-closed clausal tableau such that each node $M$ of the deduction tree can be mapped to a node $N$ of the tableau, where the partial interpolant pi $(M)$ associated with $M$ according to Huang's method is syntactically identical to the value of ipol $(N)$ as specified in Definition 6. The root of the deduction tree, labeled with $\square$, whose partial interpolant is the overall interpolation result, is mapped there to the root of the clausal tableau.

We now show this simulation for Huang's method (in the special case where it is applied to propositional formulas) in detail. To specify Huang's method, we use the "color-based"

7 That semantic trees and tree resolution can simulate each other polynomially has been shown already in [69], where also relationships to many other propositional systems are investigated. 
terminology, which is adequate here since we only consider propositional interpolation, it facilitates the incorporation of merging duplicate literals into resolution, and the objective is to compute only Craig (in contrast to Craig-Lyndon) interpolants.

Definition 11 (Huang's Partial Interpolant for Propositional Inputs) Let $F, G$ be propositional clausal formulas with no common clause and such that $F \wedge G$ is unsatisfiable. Call an atom F-colored if it occurs in $F$ but not in $G$, G-colored if it occurs in $G$ but not in $F$, and transparent if it occurs in both $F$ and $G$. For all nodes $M$ of a deduction tree for $F \wedge G$ define the partial interpolant associated with $M$ according to Huang's method $\mathrm{pi}(M)$ as follows:

(i) If $M$ is a leaf and its clause is in $F$, then $\mathrm{pi}(M) \stackrel{\text { def }}{=} \perp$.

(ii) If $M$ is a leaf and its clause is in $G$, then $\operatorname{pi}(M) \stackrel{\text { def }}{=} \mathrm{T}$.

(iii) If $M$ is an inner node with children $M_{1}, M_{2}$ and its clause is a resolvent of the clauses of $M_{1}$ and $M_{2}$ upon $A$ in the clause of $M_{1}$ and $\neg A$ in the clause of $M_{2}$, then

(a) If $A$ is F-colored, then $\operatorname{pi}(M) \stackrel{\text { def }}{=} \operatorname{pi}\left(M_{1}\right) \vee \operatorname{pi}\left(M_{2}\right)$.

(b) If $A$ is G-colored, then $\mathrm{pi}(M) \stackrel{\text { def }}{=} \mathrm{pi}\left(M_{1}\right) \wedge \mathrm{pi}\left(M_{2}\right)$.

(c) If $A$ is transparent, then $\mathrm{pi}(M) \stackrel{\text { def }}{=}\left(\operatorname{pi}\left(M_{1}\right) \wedge \neg A\right) \vee\left(A \wedge \mathrm{pi}\left(M_{2}\right)\right)$.

If $M_{0}$ is the root of a deduction tree for $F \wedge G$ as specified in the preconditions of Definition 11, then $\operatorname{pi}\left(M_{0}\right)$ is a Craig interpolant of $F$ and $\neg G$ [36,13], which can be shown via the invariants $\left(a^{\prime}\right)$ and $\left(b^{\prime}\right)$. For the case (iii).(c) in Definition 11 we have, compared to [36], flipped the sides of the first disjunct to achieve an exact syntactic correspondence with our clausal tableaux encoding. The original formula reads: $\left(\neg A \wedge \mathrm{pi}\left(M_{1}\right)\right) \vee\left(A \wedge \operatorname{pi}\left(M_{2}\right)\right)$.

Theorem 12 (Simulation of Huang's Method for Propositional Inputs with Clausal Tableaux) Let $F, G$ be propositional clausal formulas with no common clause and such that $F \wedge G$ is unsatisfiable. Let CUTS $\stackrel{\text { def }}{=} \bigwedge_{A \in \mathcal{P}_{\text {red }(F)}}(\neg A \vee A)$ and $C U T S_{\mathrm{G}} \stackrel{\text { def }}{=} \bigwedge_{A \in \mathcal{P}_{\text {red }(G)}}(\neg A \vee$ $A)$. Then there is a linear-time algorithm that computes from any deduction tree for $F \wedge G$ a two-sided leaf-closed clausal tableau for $F \wedge C U T S_{\mathrm{F}}$ and $G \wedge C U T S_{\mathrm{G}}$ with the property that if $M_{0}$ is the root of the deduction tree and $N_{0}$ is the root of the tableau, then

$$
\operatorname{pi}\left(M_{0}\right)=\operatorname{ipol}\left(N_{0}\right) .
$$

Proof (Sketch) Assume a given deduction tree for $F \wedge G$ and let $M_{0}$ be its root. We specify a function ct that maps each node $M$ of the deduction tree to a node $N=\operatorname{ct}(M)$ of a leaf-closed two-sided clausal tableau for $F \wedge C U T S_{\mathrm{F}}$ and $G \wedge C U T S_{\mathrm{G}}$ whose root is $\operatorname{ct}\left(M_{0}\right)$. It can be verified that for all nodes $M$ of the deduction tree it holds that

$$
\operatorname{pi}(M)=\operatorname{ipol}(\operatorname{ct}(M)) \text {. }
$$

It follows that $\operatorname{ct}\left(M_{0}\right)$ has all the properties in the claim, since $(*)$ implies

$$
\operatorname{pi}\left(M_{0}\right)=\operatorname{ipol}\left(\operatorname{ct}\left(M_{0}\right)\right) .
$$

The specification of ct below characterizes structural properties as well as the side and literal labels of the tableau nodes with the same case distinctions as the definition of Huang's method (Definition 11). It is not difficult to derive from ct a linear-time procedure that constructs a clausal tableau which meets the specified characteristics by a traversal of the deduction tree.

(i) If $M$ is a leaf and its clause $C=L_{1} \vee \ldots \vee L_{n}$ is in $F$, then $\operatorname{ct}(M)$ is a tableau node $N$ with children that are leaves, have side $F$, target nodes with the same side, and literals such that clause $(N)=C$. (That target nodes with the required side exist is discussed below.) The value of $\operatorname{ct}(M)$ is then the root of a tableau that can be depicted as follows: 


$$
>_{L_{1}}^{\cdot} \succ_{L_{n}}
$$

(ii) If $M$ is a leaf and its clause $C=L_{1} \vee \ldots \vee L_{n}$ is in $G$, then $\operatorname{ct}(M)$ is a tableau node $N$ with children that are leaves, have side $\mathrm{G}$, target nodes with the same side, and literals such that clause $(N)=C$. (That target nodes with the required side exist follows analogously to case (i), for which this is discussed below.) The value of $\operatorname{ct}(M)$ is then the root of a tableau that can be depicted as follows:<smiles>[3H]C[3H]</smiles>

(iii) If $M$ is an inner node with children $M_{1}, M_{2}$ and its clause is a resolvent of the clauses of $M_{1}$ and $M_{2}$ upon $A$ in the clause of $M_{1}$ and $\neg A$ in the clause of $M_{2}$, then

(a) If $A$ is F-colored, then $\operatorname{ct}(M)$ is a tableau node $N$ with $\operatorname{ct}\left(M_{1}\right)$ and $\operatorname{ct}\left(M_{2}\right)$ as children, which have side $\mathrm{F}$ and literals $\neg A$ and $A$, respectively. The value of $\operatorname{ct}(M)$ is then the root of a tableau that can be depicted as follows:

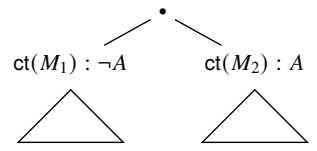

(b) If $A$ is G-colored, then $\operatorname{ct}(M)$ is a tableau node $N$ with $\operatorname{ct}\left(M_{1}\right)$ and $\operatorname{ct}\left(M_{2}\right)$ as children, which have side $\mathrm{G}$ and literals $\neg A$ and $A$, respectively. The value of $\operatorname{ct}(M)$ is then the root of a tableau that can be depicted as follows:

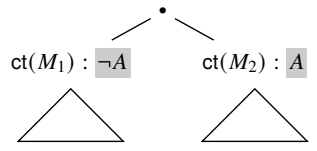

(c) If $A$ is transparent, then $\operatorname{ct}(M)$ is a tableau node $N$ with two children $N^{\prime}, N^{\prime \prime}$ with side $\mathrm{F}$ and literals $\neg A$ and $A$, respectively. The children of $N^{\prime} \operatorname{are} \operatorname{ct}\left(M_{1}\right)$ and a leaf node, with side $\mathrm{G}$ and literals $\neg A$ and $A$, respectively. The target of the leaf node is its parent $N^{\prime}$. The children of $N^{\prime \prime}$ are a leaf node and $\operatorname{ct}\left(M_{2}\right)$, with side $\mathrm{G}$ and literals $\neg A$ and $A$, respectively. The target of the leaf node is its parent $N^{\prime \prime}$. The value of $\operatorname{ct}(M)$ is then the root of a tableau that can be depicted as follows:

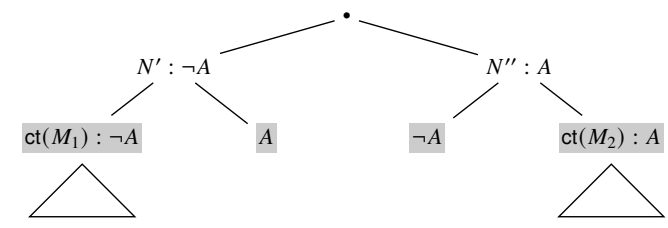

Observe that the tableaux rooted at $\operatorname{ct}\left(M_{0}\right)$ is in cut normal form, with side labelings according to the cases (i)-(iii). For any leaf with label $L_{i}$ introduced according to case (i) there exists a target with the same side $\mathrm{F}$, which can be shown as follows: Since $L_{i}$ is in a clause in $F$, its atom must be F-colored or transparent. If it is F-colored, its target must be one of the nodes with literals $\neg A$ or $A$ according to case (iii).(a), which have side $\mathrm{F}$. If it is transparent, its target must be one of the nodes with literals $\neg A$ or $A$ according to case (iii).(c). In this case, nodes with literal $\neg A$ or $A$, respectively, are present as ancestors with each side, $\mathrm{F}$ and $\mathrm{G}$, and the one with side $F$ can be selected as target. This argumentation for leaves introduced according to case (i) applies analogously to case (ii). 
Equation $(*)$ can be verified on the basis of the following observations about tableaux obtained as values of ct: Case (iii).(c) specifies two leaves with side $\mathrm{G}$ whose targets, which are their parents, have side F. By the definition of ipol, the literals of these parents enter the constructed interpolant. In fact, these are the only leaves of the tableau whose target nodes have different side, because leaves according to cases (i) and (ii) always have targets with their own side.

The following example illustrates the simulation.

Example 13 (Simulation of Huang's Method by Clausal Tableaux) Let $F=p \wedge(\neg p \vee q)$ and let $G=(\neg q \vee r) \wedge \neg$. Figure 5 shows a deduction tree for $F \wedge G$, displayed as a conventional tree, instead of the usual upside down presentation of deduction trees [20]. Nodes are labeled by clauses. Transparency is indicated by a frame and color G by gray background. The remaining symbols have color $F$. The values of pi as determined by Huang's method are annotated in brackets, where values are shown as specified in Definition 11 except that truthvalue simplification (see footnote 5) is applied. Figure 6 shows the corresponding leaf-closed two-sided clausal tableau that simulates Huang's method, related by the ct mapping. Side G is indicated by gray background. The values of ipol are annotated in brackets, again after truth value simplification. They are underlined for nodes that are values of ct for some node of the resolution tree. The target labels of the leaves are symbolized by arrows. (Observe in particular that the leaf with literal $q$ has as target not its closest ancestor with literal $\neg q$ because the side of that ancestor is not $F$. ) The clausal tableau is for $F \wedge(\neg p \vee p) \wedge(\neg q \vee q)$ and $G \wedge(\neg q \vee q) \wedge(\neg r \vee r)$.

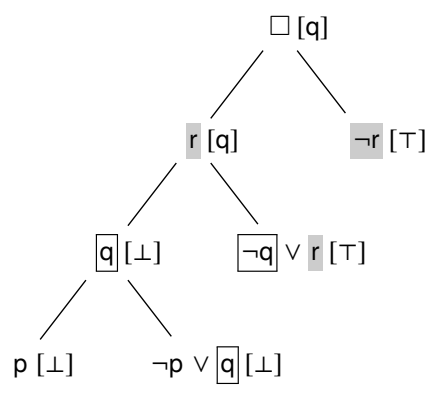

Fig. 5: Resolution deduction tree and partial interpolant annotation according to Huang's method.

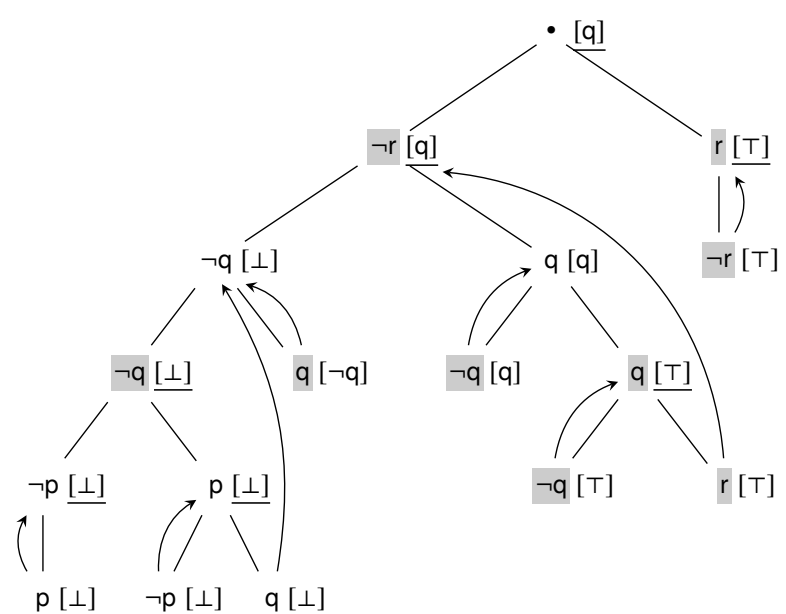

Fig. 6: Simulating Huang's method with a clausal tableau.

As already indicated, the mapping ct is a modification of the known conversion of deduction trees into clausal tableaux in cut normal form that is used to show that clausal tableaux with atomic cut and semantic trees can linearly simulate each other, which, since semantic trees can linearly simulate tree resolution, implies that tableaux with atomic cut can linearly simulate tree resolution [49, Chapter 7]. Differences are the additional labeling of nodes with a side and the introduction of stacked cuts upon the same atom, but with different side labels for the case (iii).(c). Through these stacked cuts the tableau is not regular [38], 
that is, different nodes on a branch are labeled with the same literal, however with different side labeling.

The HKPYM interpolation system shown in [12,13] is similar to Huang's method for the propositional case, but had also been discovered and analyzed independently by several other authors (see [13] for references, the name HKPYM represents initials of these authors, with $H$ for Huang). It differs from Huang's method in that the case (iii).(c) of Definition 11 is replaced with $\mathrm{pi}(M) \stackrel{\text { def }}{=}\left(A \vee \mathrm{pi}\left(M_{1}\right)\right) \wedge\left(\neg A \vee \mathrm{pi}\left(M_{2}\right)\right)$. If we flip the sides of the first conjunct and take $\mathrm{pi}(M) \stackrel{\text { def }}{=}\left(\mathrm{pi}\left(M_{1}\right) \vee A\right) \wedge\left(\neg A \vee \mathrm{pi}\left(M_{2}\right)\right)$ instead, that method can also be simulated with clausal tableaux such that syntactically identical interpolants are obtained: We just have to modify case (iii).(c) of the definition of ct such that the upper cut has side G and the two lower cuts have side F.

Optimized Huang [8] is a variant of Huang's method for computing Craig-Lyndon interpolants which specializes the provisional interpolation system of [14, Def. 15]. It uses a labeling with respect to literal occurrences, as discussed on page 8 . The case corresponding to (iii).(c) of Definition 11 is split into subcases depending on this labeling, for example (considering the algorithm just for propositional inputs) $\mathrm{pi}(M) \stackrel{\text { def }}{=} \mathrm{pi}\left(M_{1}\right) \vee\left(A \wedge \mathrm{pi}\left(M_{2}\right)\right){ }^{8}$ It is straightforward to adapt ct to these cases, as depicted in Figure 7 for the example case: ${ }^{9}$

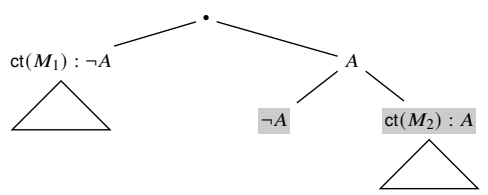

Fig. 7: The case of optimized Huang where $\mathrm{pi}(M) \stackrel{\text { def }}{=} \mathrm{pi}\left(M_{1}\right) \vee\left(A \wedge \mathrm{pi}\left(M_{2}\right)\right)$ in tableau simulation.

The encoding of deduction trees into clausal tableaux for interpolant computation with ipol suggests further variants of interpolant extraction from resolution proofs, waiting for exploration or providing a simulation of further known interpolation methods for resolution proofs. It is, for example, possible to translate the case (iii).(c) not into stacked cuts (i.e., a cut with side $F$ where cuts with side $G$ are attached to its children) as for the simulation of Huang's method but like cases (iii).(a) and (iii).(b) into a single cut, where cuts upon a colored symbol receive the corresponding side label, and cuts upon a transparent symbol receive an arbitrary side label. The resulting tableau is then also a leaf-closed two-sided clausal tableau for $F \wedge C U T S_{\mathrm{F}}$ and $G \wedge C U T S_{\mathrm{G}}$ that permits to apply ipol. It is not difficult to verify that if side $G$ is chosen for all cuts upon transparent symbols in this deduction tree translation, then a linear simulation of McMillan's method [58,60], presented as interpolation system $M M$ in [13], is obtained. Here is an example.

8 To take account of merging duplicate literals, the method as presented in [8] has to be supplemented by the explicit consideration of factoring steps with the dedicated assignment of partial interpolants from [14, Def. 15], or, alternatively, it must be possible to label an occurrence with both provenance values at the same time, as noted in [36], and retaining Huang's original definition for case (iii).(c) for the subcase where the resolution step is upon two literals which each have both provenances. As an example where merging is necessary consider $F=(p \vee q) \wedge(\neg p \vee r)$ and $G=(p \vee \neg q) \wedge(\neg p \vee \neg r)$.

9 The argument in the proof of Theorem 12 to show that in case (i) for any leaf with label $L_{i}$ there exists a target with the same side $F$ can also be applied here, but the analogy for case (ii) has to be shown differently. It follows since in case (ii) a leaf with label $L_{i}$ must have an ancestor with complementary literal that was introduced by the encoding of a resolution step upon $L_{i}$ and its complement. If the label of the involved occurrence of the complement is $F$, then the encoding of the resolution step would result in two successive nodes with the complement of $L_{i}$, one with side F and one with side $G$. 
Example 14 (Simulation of McMillan's Method by Clausal Tableaux) This example is analogous to Example 13, but for McMillan's instead of Huang's method. The deduction trees in Figure 5 and 8 differ only in the annotated partial interpolants. For McMillan's method they are according to [13, Def. 21]. In the tableau translation for Huang's method (Figure 6), below the left child of the root "stacked cuts" upon the transparent atom q are inserted, according to case (iii).(c) of Definition 11. For the simulation of McMillan's method shown in Figure 9, only a single cut upon $q$ is inserted at this position. Its side is G, which is chosen for all cuts upon transparent atoms in the simulation of McMillan's method. In the simulation, the target of a leaf is always closest ancestor with complementary literal.

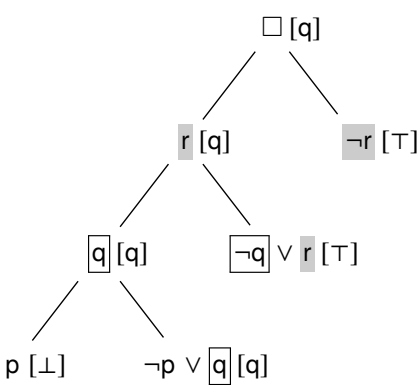

Fig. 8: Resolution deduction tree and partial interpolant annotation according to McMillan's method.

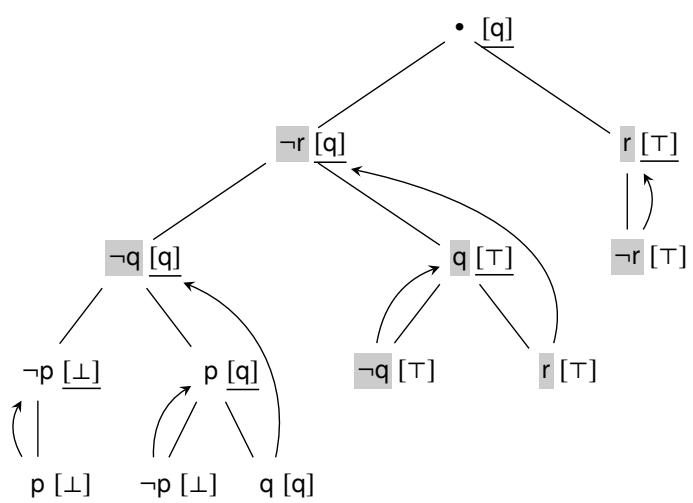

Fig. 9: Simulating McMillan's method with a clausal tableau.

\section{Lifting Ground Interpolants - Additional Notation}

We now turn to the computation of first-order interpolants, beyond the ground case. As outlined in Sect. 1.2, we follow a two-stage approach. The second stage, interpolant lifting, is discussed in the following Sects. 7-9. Related work on interpolant lifting will be discussed in Sect. 10.1. As interpolant lifting concerns terms, we need additional notation that was not relevant in the previous sections.

The Subterm Relationship. We write $s \triangleleft t$ to express that $s$ is a strict subterm of $t .{ }^{10}$

Terms with Outermost Function Symbol in a Given Set. If $\mathcal{S}$ is a set of function symbols, then a term whose outermost function symbol is in $\mathcal{S}$ is called an $\mathcal{S}$-term. The plural form $\mathcal{S}$-terms is also used to denote the set of all $\mathcal{S}$-terms.

Substitutions. A substitution is a mapping from variables to terms which is almost everywhere equal to identity. If $\sigma$ is a substitution, then the domain of $\sigma$ is the set of variables $\operatorname{Dom}(\sigma) \stackrel{\text { def }}{=}\{x \mid x \sigma \neq x\}$, the range of $\sigma$ is $\operatorname{Rng}(\sigma) \stackrel{\text { def }}{=} \bigcup_{x \in \mathcal{D} o m(\sigma)}\{x \sigma\}$, and the restriction of $\sigma$ to a set $\mathcal{X}$ of variables, denoted by $\left.\sigma\right|_{\mathcal{X}}$, is the substitution which is equal to the identity everywhere except over $\mathcal{X} \cap \operatorname{Dom}(\sigma)$, where it is equal to $\sigma$. The identity substitution is denoted by $\varepsilon$. We write the set $\operatorname{Var}(\mathcal{R} n g(\sigma))$ of variables in the range of substitution $\sigma$ also

10 The symbol $\triangleleft$ is an adaptation of $t \unrhd s$ for $s$ is a subterm of $t$, common in the literature on term rewriting [27]. We use it in the strict version and in flipped direction because it is then in direct correspondence with ordering constraints on terms that determine the order of quantifications in our Theorem 21. 
as $\mathcal{V} R n g(\sigma)$. If all members of the range of a substitution are ground, then the substitution is called a ground substitution.

A substitution can be represented as a function by a set of assignments to the variables in its domain, e.g., $\left\{x_{1} \mapsto t_{1}, \ldots, x_{n} \mapsto t_{n}\right\}$. The application of a substitution $\sigma$ to a term or a formula $E$ is written as $E \sigma, E \sigma$ is called an instance of $E$ and $E$ is said to subsume $E \sigma$. Composition of substitutions is written as juxtaposition. Hence, if $\sigma$ and $\gamma$ are both substitutions, then $E \sigma \gamma$ stands for $(E \sigma) \gamma$.

Maximal Occurrences and Inverse Application of an Injective Substitution. An occurrence of a member $t$ of a set $\mathcal{T}$ of terms in a term or a formula $E$ is called $\mathcal{T}$-maximal if it is not within an occurrence of another member of $\mathcal{T}$. If $\sigma$ is an injective substitution and $E$ is a term or a formula, then $E\left\langle\sigma^{-1}\right\rangle$ denotes $E$ with all $\mathcal{R} n g(\sigma)$-maximal occurrences of terms $t \in \mathcal{R} n g(\sigma)$ replaced by the variable that is mapped by $\sigma$ to $t$. As an example let $\sigma=\{x \mapsto \mathrm{f}(\mathrm{a}), y \mapsto \mathrm{g}(\mathrm{f}(\mathrm{a}))\}$. Then

$$
\mathrm{p}(\mathrm{h}(\mathrm{f}(\mathrm{a}), \mathrm{g}(\mathrm{f}(\mathrm{a}))))\left\langle\sigma^{-1}\right\rangle=\mathrm{p}(\mathrm{h}(x, y)) .
$$

The inverse application of an injective substitution $\sigma$ to a term or formula $E$ can be described operationally as follows: (1) construct the inverse of $\sigma$, which is $\{\mathrm{f}(\mathrm{a}) \mapsto x, \mathrm{~g}(\mathrm{f}(\mathrm{a})) \mapsto y\}$ in our example. As can be seen with the example, the inverse is in general not a substitution because arbitrary terms and not just variables can be in its domain. (2) Order the members of the inverse into a sequence of pairs such that whenever for two pairs $t \mapsto v$ and $t^{\prime} \mapsto v^{\prime}$ it holds that $t^{\prime} \triangleleft t$, then $t \mapsto v$ appears to the left of $t^{\prime} \mapsto v^{\prime}$. For the example we obtain $\langle\mathrm{g}(\mathrm{f}(\mathrm{a})) \mapsto y, \mathrm{f}(\mathrm{a}) \mapsto x\rangle$. (3) For each member $t \mapsto v$ of the sequence, proceeding from left to right, replace all occurrences of $t$ in $E$ with $v$. In our example we first obtain $\mathrm{p}(\mathrm{h}(\mathrm{f}(\mathrm{a}), y))$ as intermediate result and then the final result $\mathrm{p}(\mathrm{h}(x, y))$. We note the following properties of inversely applied substitutions.

Proposition 15 (Properties of Inverse Substitutions) Let $\sigma$ be an injective substitution. Then:

(i) If $E$ is a term or a quantifier-free formula such that $\operatorname{Dom}(\sigma) \cap \operatorname{Var}(E)=\emptyset$ then $E\left\langle\sigma^{-1}\right\rangle \sigma=E$.

(ii) If $F, G$ are quantifier-free formulas such that $F \models G$ then $F\left\langle\sigma^{-1}\right\rangle \vDash G\left\langle\sigma^{-1}\right\rangle$.

Proof (Sketch) (15.i) Easy to see. (15.ii) Recall that we only consider formulas without equality. A quantifier-free formula is valid if and only if it is propositionally valid, that is, the propositional formula obtained by taking each atom $p\left(t_{1}, \ldots, t_{n}\right)$ as name of a propositional variable is valid. If $A$ is an atom occurring in a quantifier-free formula, then the inverse application of an injective substitution $\sigma$ effects that either no occurrence of $A$ is replaced or all occurrences of $A$ are replaced by the same atom $A\left\langle\sigma^{-1}\right\rangle$. This implies that the inverse application of an injective substitution to a valid quantifier-free formula yields again a valid quantifier-free formula: Any proof that shows the propositional validity of the original formula can be modified to a proof of the propositional validity of the formula after the inverse application by just replacing the propositional variables that name replaced atoms $A$ with the propositional variables that name the respective replacement atoms $A\left\langle\sigma^{-1}\right\rangle$. (This holds independently from whether names of some of the replacing atoms $A\left\langle\sigma^{-1}\right\rangle$ were already used in the original proof.) Assuming that $F$ and $G$ are quantifier-free formulas such that $F \vDash G$ we can conclude that $F \rightarrow G$ is a valid quantifier-formula, hence $(F \rightarrow G)\left\langle\sigma^{-1}\right\rangle$, which identical to $F\left\langle\sigma^{-1}\right\rangle \rightarrow G\left\langle\sigma^{-1}\right\rangle$, is valid, hence $F\left\langle\sigma^{-1}\right\rangle \mid=G\left\langle\sigma^{-1}\right\rangle$. 


\section{Interpolant Lifting Bases and Statement of the Interpolant Lifting Theorem}

As already indicated informally, the lifting to first-order interpolants can be specified on the basis of Craig-Lyndon interpolants of ground formulas that are in a certain relationship to the original first-order interpolation inputs, where the arguments for the correctness of the lifting transformation are independent of a particular calculus. Instead, the correctness proof is based on second-order Skolemization and Herbrand's theorem. The concept of interpolant lifting base, defined below, provides an interface between formula constructions by secondorder Skolemization and quantifier expansion according to Herbrand's theorem on the one hand and those abstract properties of the constructed formulas that are needed to justify interpolant lifting on the other hand. It should be noted, that these formula constructions are applied only to prove the correctness of interpolant lifting, without implying the necessity to actually perform them at interpolant computation. Thus, the correctness of interpolant lifting is shown via the existence of certain constructible formulas.

An interpolant lifting base gathers certain components and relates them by constraints. These components are the original interpolation inputs $F, G$, intermediate formulas $F_{\mathrm{EXP}}, G_{\mathrm{EXP}}$ (constructible from $F$ and $G$ by Skolemization and expansion according to Herbrand's theorem), sets of function symbols $\mathcal{F}, \mathcal{G}$ (symbols that are not permitted to occur in the lifted interpolant and include the Skolem functions in $F_{\mathrm{EXP}}$ and $G_{\mathrm{EXP}}$ ), a ground substitution $\eta_{\mathrm{EXP}}$ whose domain is the set of the variables occurring in $F_{\mathrm{EXP}}$ and $G_{\mathrm{EXP}}$, and a ground interpolant $H_{\mathrm{GRD}}$ of the ground formulas $F_{\mathrm{EXP}} \eta_{\mathrm{EXP}}$ and $G_{\mathrm{EXP}} \eta_{\mathrm{EXP}}$. In the lifting base these components are arranged as a tuple such that the components $F, G, \mathcal{F}, \mathcal{G}, H_{\mathrm{GRD}}$, which are those that need to be materialized at the interpolant lifting formula transformation, precede the other ones $F_{\mathrm{EXP}}, G_{\mathrm{EXP}}, \eta_{\mathrm{EXP}}$. The following definition makes interpolant lifting base precise.

Definition 16 (Interpolant Lifting Base) An interpolant lifting base (briefly lifting base) is a tuple

$$
\left\langle F, G, \mathcal{F}, \mathcal{G}, H_{\mathrm{GRD}}, F_{\mathrm{EXP}}, G_{\mathrm{EXP}}, \eta_{\mathrm{EXP}}\right\rangle,
$$

where $F, G$ are first-order sentences, $\mathcal{F}, \mathcal{G}$ are disjoint sets of function symbols, $H_{\text {GRD }}$ (the subscript GRD suggesting ground) is a ground formula, $F_{\mathrm{EXP}}, G_{\mathrm{EXP}}$ (the subscript EXP suggesting expansion) are quantifier-free formulas and $\eta_{\mathrm{EXP}}$ is a ground substitution such that:

(i) $F \mid=\exists \mathcal{F} \forall \mathcal{U} F_{\mathrm{EXP}}$ where $\mathcal{U}=\operatorname{Var}\left(F_{\mathrm{EXP}}\right)$.

(ii) $\operatorname{Pred}^{ \pm}\left(F_{\mathrm{EXP}}\right) \subseteq \mathcal{P r e d}^{ \pm}(F)$.

(iii) $\mathcal{F} u n\left(F_{\mathrm{EXP}}\right) \subseteq(\mathcal{F} u n(F) \cap \mathcal{F} u n(G)) \cup \mathcal{F}$.

(iv) $\mathcal{F} u n(F) \cap \mathcal{G}=\emptyset$.

(v) $\operatorname{Dom}\left(\eta_{\mathrm{EXP}}\right)=\operatorname{Var}\left(F_{\mathrm{EXP}}\right) \cup \mathcal{V a r}\left(G_{\mathrm{EXP}}\right)$.

(vi) $\mathcal{F} u n\left(\mathcal{R} n g\left(\eta_{\mathrm{EXP}}\right)\right) \subseteq \mathcal{F} u n\left(F_{\mathrm{EXP}}\right) \cup \mathcal{F} u n\left(G_{\mathrm{EXP}}\right) \cup\left\{\mathrm{c}_{0}\right\}$, where $\mathrm{c}_{0}$ is a constant in $\mathcal{F} \cup \mathcal{G}$.

(vii) $H_{\mathrm{GRD}}$ is a Craig-Lyndon interpolant of $F_{\mathrm{EXP}} \eta_{\mathrm{EXP}}$ and $G_{\mathrm{EXP}} \eta_{\mathrm{EXP}}$.

We now consider the constructive aspect of interpolant lifting bases, showing with Proposition 19 that for given first-order sentences $F, G$ such that $F \vDash G$ a lifting base exists. The proof shows this constructively, providing intuitions that flesh out the items abstractly related by constraints in Definition 16. Further intuition might be provided with Procedure 23 and Example 20 below. Proposition 19 is preceded by two auxiliary propositions about Skolemization and Herbrand's theorem. 
Proposition 17 (Second-Order Skolemization) Let $F$ be a formula. Assume that $x_{1}, \ldots, x_{n}$, $y$ are variables that do not occur bound in $F$ and that $f$ is an n-ary function symbol that does not occur in $F$. Then $\forall x_{1} \ldots \forall x_{n} \exists y F \equiv \exists f \forall x_{1} \ldots \forall x_{n} F\left\{y \mapsto f\left(x_{1}, \ldots, x_{n}\right)\right\}$.

If $f$ in Proposition 17 is nullary, that is, if $f$ is a Skolem constant, then the quantification upon $f$ is first-order quantification upon $f$ in the role of a variable. Otherwise, the quantification is second-order quantification upon a function symbol. We need second-order quantification to capture the semantics of Skolemization, not just the commonly used preservation of satisfiability and unsatisfiability. However, we use second-order quantification only in contexts where actual reasoning in second-order logic is not required.

The following proposition expresses Herbrand's theorem as used in automated theorem proving in a form that applies to conjunctions of universal first-order sentences.

Proposition 18 (A Form of Herbrand's Theorem) A first-order sentence $S$ of the form $\forall X_{1} C_{1} \wedge \ldots \wedge \forall \mathcal{X}_{n} C_{n}$, where $\mathcal{X}_{1}, \ldots, \mathcal{X}_{n}$ are finite sets of variables and $C_{1}, \ldots, C_{n}$ are quantifier-free formulas is unsatisfiable if and only if there exists a finite unsatisfiable conjunction of ground instances of formulas $C_{i}$ with $i \in\{1, \ldots, n\}$, obtained by instantiating with terms formed from functions occurring in $S$ (and, if no constant occurs in $S$, a fresh constant). ${ }^{11}$

We can now claim the existence of an interpolant base for sentences $F, G$ such that $F \models G$ and prove it with a construction.

Proposition 19 (Existence of an Interpolant Lifting Base) If $F, G$ are first-order sentences such that $F \vDash G$, then there exists an interpolant lifting base

$$
\left\langle F, G, \mathcal{F}, \mathcal{G}, H_{G R D}, F_{E X P}, G_{E X P}, \eta_{E X P}\right\rangle .
$$

Proof Given $F \mid=G$, the remaining components of an interpolant lifting base can be constructed as follows: Apply conversion to prenex form and Skolemization (according to Proposition 17) independently to $F$ and to $\neg G$, to obtain disjoint sets of fresh Skolem functions $\mathcal{F}^{\prime}, \mathcal{G}^{\prime}$, quantifier-free formulas $F^{\prime}, G^{\prime}$, and sets $\mathcal{U}^{\prime}=\mathcal{V} \operatorname{Var}\left(F^{\prime}\right), \mathcal{V}^{\prime}=\operatorname{Var}\left(G^{\prime}\right)$ of variables such that:

(1) $F \equiv \exists \mathcal{F}^{\prime} \forall \mathcal{U}^{\prime} F^{\prime}$.

(1') $\neg G \equiv \exists \mathcal{G}^{\prime} \forall \mathcal{V}^{\prime} G^{\prime}$.

(2) $\mathcal{V}_{o c^{ \pm}}\left(F^{\prime}\right) \subseteq \mathcal{V}^{\prime} c^{ \pm}(F) \cup \mathcal{F}^{\prime}$.

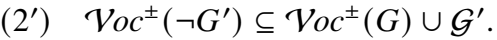

(3) $\forall \mathcal{U}^{\prime} \forall \mathcal{V}^{\prime}\left(F^{\prime} \wedge G^{\prime}\right) \vDash \perp$

If there is a constant occurring in $F^{\prime} \wedge G^{\prime}$, then let $\mathrm{c}_{0}$ be such a constant and let $C \stackrel{\text { def }}{=} \emptyset$, else let $\mathrm{c}_{0}$ be a fresh constant and let $C \stackrel{\text { def }}{=}\left\{\mathrm{c}_{0}\right\}$. From Herbrand's theorem (Proposition 18) and (3) it follows that there exist quantifier-free formulas $F_{\mathrm{EXP}}, G_{\mathrm{EXP}}$ and a ground substitution $\eta_{\mathrm{EXP}}$ such that:

(4) $\operatorname{Dom}\left(\eta_{\mathrm{EXP}}\right)=\operatorname{Var}\left(F_{\mathrm{EXP}}\right) \cup \operatorname{Var}\left(G_{\mathrm{EXP}}\right)$.

(5) $\mathcal{F} u n\left(\mathcal{R} n g\left(\eta_{\mathrm{EXP}}\right)\right) \subseteq \mathcal{F} u n\left(F_{\mathrm{EXP}}\right) \cup \mathcal{F} u n\left(G_{\mathrm{EXP}}\right) \cup\left\{\mathrm{c}_{0}\right\}$.

(6) $F_{\mathrm{EXP}} \eta_{\mathrm{EXP}} \wedge \neg G_{\mathrm{EXP}} \eta_{\mathrm{EXP}}=\perp$.

(7) $\forall \mathcal{U}^{\prime} F^{\prime}=\forall \mathcal{U} F_{\mathrm{EXP}}$, where $\mathcal{U}=\operatorname{Var}\left(F_{\mathrm{EXP}}\right)$

( $\left.7^{\prime}\right) \quad \forall \mathcal{V}^{\prime} G^{\prime}=\forall \mathcal{V} \neg G_{\mathrm{EXP}}$, where $\mathcal{V}=\mathcal{V} \operatorname{Var}\left(G_{\mathrm{EXP}}\right)$

(8) $\mathcal{V} c^{ \pm}\left(F_{\mathrm{EXP}}\right) \subseteq \mathcal{V} \operatorname{Voc}^{ \pm}\left(F^{\prime}\right)$.

$\left(8^{\prime}\right) \operatorname{Voc}^{ \pm}\left(G_{\mathrm{EXP}}\right) \subseteq \mathcal{V}^{ \pm} c^{ \pm}\left(\neg G^{\prime}\right)$.

${ }_{11} C_{1}, \ldots, C_{n}$ can be arbitrary quantifier-free formulas, with clauses as a special case. 
Steps (7), $\left(7^{\prime}\right),(8)$ and $\left(8^{\prime}\right)$ hold since Proposition 18 justifies the construction of $F_{\text {EXP }}$ as a conjunction of variants of $F^{\prime}$ (that is, formulas obtained from $F^{\prime}$ by systematically renaming variables) and the construction of $G_{\mathrm{EXP}}$ as the negation of a conjunction of variants of $G^{\prime} .^{12}$ Construct the sets $\mathcal{F}$ and $\mathcal{G}$ of function symbols according to the following definitions:

(9) $\mathcal{F} \stackrel{\text { def }}{=} \mathcal{F}^{\prime} \cup(\mathcal{F} u n(F) \backslash \mathcal{F} u n(G)) \cup C$.

$\left(9^{\prime}\right) \quad \mathcal{G} \stackrel{\text { def }}{=} \mathcal{G}^{\prime} \cup(\mathcal{F} u n(G) \backslash \mathcal{F} u n(F))$.

That is, $\mathcal{F}$ and $\mathcal{G}$ extend the sets of Skolem functions for $F$ and $\neg G$ by the functions occurring in $F$ but not in $G$ and vice versa, respectively. In addition, if a fresh symbols is needed as constant $\mathrm{c}_{0}$, it is added to $\mathcal{F}$.

That the constructed elements match conditions (i), (ii), (iii) and (iv) of Definition 16 can be shown in the following steps, explained below, where the respective conditions are annotated on the right. Conditions (i'), (ii'), (iii') and (iv') of Definition 16 can be derived in straightforward analogy.

(10) $F \mid=\exists \mathcal{F} \forall \mathcal{U} F_{\mathrm{EXP}}$, where $\mathcal{U}=\operatorname{Var}\left(F_{\mathrm{EXP}}\right)$.

Def. 16.(i)

(11) $\operatorname{Pred}^{ \pm}\left(F_{\mathrm{EXP}}\right) \subseteq \mathcal{P r e d}^{ \pm}(F)$.

Def. 16.(ii)

(12) $\mathcal{F} u n\left(F_{\mathrm{EXP}}\right) \subseteq \mathcal{F} u n(F) \cup \mathcal{F}^{\prime}$.

(13) $\mathcal{F} u n\left(F_{\mathrm{EXP}}\right) \subseteq \mathcal{F} u n(F) \cup \mathcal{F}^{\prime} \cup C$.

(14) $\mathcal{F} u n(F) \cup \mathcal{F}^{\prime} \cup C$

$=(\mathcal{F} u n(F) \cap \mathcal{F} u n(G)) \cup(\mathcal{F u n}(F) \backslash \mathcal{F} u n(G)) \cup \mathcal{F}^{\prime} \cup C$

$=(\mathcal{F} u n(F) \cap \mathcal{F} u n(G)) \cup \mathcal{F}$.

(15) $\operatorname{Fun}\left(F_{\mathrm{EXP}}\right) \subseteq(\mathcal{F} u n(F) \cap \mathcal{F} u n(G)) \cup \mathcal{F}$. Def. 16.(iii)

(16) $\mathcal{F} u n(F) \cap \mathcal{G}=\emptyset$. Def. 16.(iv)

Step (10) follow from (1) and (7). Steps (11) and (12) both follow from (8) and (2). Step (13) follows from (12). Step (14) follows from general properties of sets and (9). Step (15) follows from (13) and (14). Step (16) follow from step $\left(9^{\prime}\right)$, since $\mathcal{G}^{\prime}$ contains only fresh symbols.

Conditions (v) and (vi) of Definition 16 have already been stated as steps (4) and (5). It remains to consider condition (vii). Step (6) can be expressed as $F_{\mathrm{EXP}} \eta_{\mathrm{EXP}}=G_{\mathrm{EXP}} \eta_{\mathrm{EXP}}$. By our ground interpolation method from Sect. 4 (or any other constructive statement of the Craig-Lyndon interpolation theorem for ground formulas) it follows that from $F_{\mathrm{EXP}} \eta_{\mathrm{EXP}}$ and $G_{\mathrm{EXP}} \eta_{\mathrm{EXP}}$ a Craig-Lyndon interpolant $H_{\mathrm{GRD}}$ of $F_{\mathrm{EXP}} \eta_{\mathrm{EXP}}$ and $G_{\mathrm{EXP}} \eta_{\mathrm{EXP}}$ can be constructed. Condition (vii) of Definition 16, which states that $H_{\mathrm{GRD}}$ is a Craig-Lyndon interpolant of $F_{\mathrm{EXP}} \eta_{\mathrm{EXP}}$ and $G_{\mathrm{EXP}} \eta_{\mathrm{EXP}}$, is then evidently satisfied.

Here are several examples of interpolant lifting bases that illustrate different aspects.

Example 20 (Interpolant Lifting Base) The following are examples of interpolant lifting bases with specific properties described as introductory comments:

(i) $\mathcal{F}$ contains a non-constant; members of $\mathcal{F}$ as well as a members of $\mathcal{G}$ occur in $H_{\text {GRD }}$ :

$$
\begin{gathered}
\left\langle F=\forall x \mathrm{p}(x, \mathrm{f}(x)), G=\exists x \mathrm{p}(\mathrm{g}, x), \mathcal{F}=\{\mathrm{f}\}, \mathcal{G}=\{\mathrm{g}\}, H_{\mathrm{GRD}}=\mathrm{p}(\mathrm{g}, \mathrm{f}(\mathrm{g})),\right. \\
\left.F_{\mathrm{EXP}}=\mathrm{p}\left(u_{1}, \mathrm{f}\left(u_{1}\right)\right), G_{\mathrm{EXP}}=\mathrm{p}\left(\mathrm{g}, u_{2}\right), \eta_{\mathrm{EXP}}=\left\{u_{1} \mapsto \mathrm{g}, u_{2} \mapsto \mathrm{f}(\mathrm{g})\right\}\right\rangle .
\end{gathered}
$$

In this example it holds that $F_{\mathrm{EXP}} \eta_{\mathrm{EXP}}=G_{\mathrm{EXP}} \eta_{\mathrm{EXP}}=H_{\mathrm{GRD}}=\mathrm{p}(\mathrm{g}, \mathrm{f}(\mathrm{g}))$.

\footnotetext{
12 In the case where $F^{\prime}$ and $G^{\prime}$ are clausal formulas, there also may exist smaller formulas $F_{\mathrm{EXP}}$ and $G_{\mathrm{EXP}}$ obtained as conjunction of variants of clauses of $F^{\prime}$ and by negating a conjunction of variants of clauses of $G^{\prime}$, respectively.
} 
(ii) A member of $\mathcal{F}$ (i.e., $\mathrm{f}_{2}$ ) is a Skolem function:

$$
\begin{aligned}
& \left\langle F=\forall x \exists y \mathrm{p}\left(x, y, \mathrm{f}_{1}\right), G=\exists x \exists y \mathrm{p}(\mathrm{g}, x, y), \mathcal{F}=\left\{\mathrm{f}_{1}, \mathrm{f}_{2}\right\}, \mathcal{G}=\{\mathrm{g}\},\right. \\
& H_{\mathrm{GRD}}=\mathrm{p}\left(\mathrm{g}, \mathrm{f}_{2}(\mathrm{~g}), \mathrm{f}_{1}\right), F_{\text {EXP }}=\mathrm{p}\left(u_{1}, \mathrm{f}_{2}\left(u_{1}\right), \mathrm{f}_{1}\right), G_{\mathrm{EXP}}=\mathrm{p}\left(\mathrm{g}, u_{2}, u_{3}\right), \\
& \left.\eta_{\mathrm{EXP}}=\left\{u_{1} \mapsto \mathrm{g}, u_{2} \mapsto \mathrm{f}_{2}(\mathrm{~g}), u_{3} \mapsto \mathrm{f}_{1}\right\}\right\rangle .
\end{aligned}
$$

In this example it holds that $F_{\mathrm{EXP}} \eta_{\mathrm{EXP}}=G_{\mathrm{EXP}} \eta_{\mathrm{EXP}}=H_{\mathrm{GRD}}=\mathrm{p}\left(\mathrm{g}, \mathrm{f}_{2}(\mathrm{~g}), \mathrm{f}_{1}\right)$.

(iii) $F_{\mathrm{EXP}}$ is a conjunction of different variants of the matrix $\mathrm{p}(x, \mathrm{f})$ of $F$ :

$$
\begin{aligned}
& \left\langle F=\forall x \mathrm{p}(x, \mathrm{f}), G=\exists x \mathrm{p}\left(\mathrm{g}_{1}, x\right) \wedge \exists x \mathrm{p}\left(\mathrm{g}_{2}, x\right), \mathcal{F}=\{\mathrm{f}\}, \mathcal{G}=\left\{\mathrm{g}_{1}, \mathrm{~g}_{2}\right\},\right. \\
& H_{\mathrm{GRD}}=\mathrm{p}\left(\mathrm{g}_{1}, \mathrm{f}\right) \wedge \mathrm{p}\left(\mathrm{g}_{2}, \mathrm{f}\right), F_{\mathrm{EXP}}=\mathrm{p}\left(u_{1}, \mathrm{f}\right) \wedge \mathrm{p}\left(u_{2}, \mathrm{f}\right), G_{\mathrm{EXP}}=\mathrm{p}\left(\mathrm{g}_{1}, u_{3}\right) \wedge \mathrm{p}\left(\mathrm{g}_{2}, u_{3}\right), \\
& \left.\eta_{\mathrm{EXP}}=\left\{u_{1} \mapsto \mathrm{g}_{1}, u_{2} \mapsto \mathrm{g}_{2}, u_{3} \mapsto \mathrm{f}\right\}\right\rangle
\end{aligned}
$$

In this example it holds that $F_{\mathrm{EXP}} \eta_{\mathrm{EXP}}=G_{\mathrm{EXP}} \eta_{\mathrm{EXP}}=H_{\mathrm{GRD}}=\mathrm{p}\left(\mathrm{g}_{1}, \mathrm{f}\right) \wedge \mathrm{p}\left(\mathrm{g}_{2}, \mathrm{f}\right)$.

(iv) The input formulas $F, G$ in this example extend those of (i) by additional literals with predicates $\mathrm{q}, \mathrm{r}$ that occur in only one of them and a second function symbol in $G$. Differently from the previous three cases, in this case the ground interpolant is not an instance of the intermediate formula $F_{\mathrm{EXP}}$. Values of $F_{\mathrm{EXP}}, G_{\mathrm{EXP}}$ and $\eta_{\mathrm{EXP}}$ with other features than shown here are also possible. For example, the variables $u_{3}, u_{4}$ could be merged with $u_{1}$, or $\eta_{\mathrm{EXP}}$ could assign $u_{3}, u_{4}$ to some other ground term. The values here have been chosen, as they are suited to illustrate further aspects in Example 28 and the discussion following Definition 29.

$$
\begin{aligned}
& \left\langle F=\forall x \mathrm{p}(x, \mathrm{f}(x)) \wedge \forall x \forall y \mathrm{q}(\mathrm{f}(x), y), G=\exists x\left(\mathrm{p}\left(\mathrm{g}_{1}, x\right) \vee \mathrm{r}\left(\mathrm{g}_{2}(x)\right)\right),\right. \\
& \mathcal{F}=\{\mathrm{f}\}, \mathcal{G}=\left\{\mathrm{g}_{1}, \mathrm{~g}_{2}\right\}, H_{\mathrm{GRD}}=\mathrm{p}\left(\mathrm{g}_{1}, \mathrm{f}\left(\mathrm{g}_{1}\right)\right), \\
& F_{\text {EXP }}=\mathrm{p}\left(u_{1}, \mathrm{f}\left(u_{1}\right)\right) \wedge \mathrm{q}\left(\mathrm{f}\left(u_{3}\right), u_{4}\right), G_{\mathrm{EXP}}=\mathrm{p}\left(\mathrm{g}_{1}, u_{2}\right) \vee \mathrm{r}\left(\mathrm{g}_{2}\left(u_{2}\right)\right), \\
& \left.\eta_{\mathrm{EXP}}=\left\{u_{1} \mapsto \mathrm{g}_{1}, u_{2} \mapsto \mathrm{f}\left(\mathrm{g}_{1}\right), u_{3} \mapsto \mathrm{g}_{2}\left(\mathrm{f}\left(\mathrm{g}_{1}\right)\right), u_{4} \mapsto \mathrm{g}_{1}\right\}\right\rangle
\end{aligned}
$$

In this example it holds that $F_{\mathrm{EXP}} \eta_{\mathrm{EXP}}=\mathrm{p}\left(\mathrm{g}_{1}, \mathrm{f}\left(\mathrm{g}_{1}\right)\right) \wedge \mathrm{q}\left(\mathrm{f}\left(\mathrm{g}_{2}\left(\mathrm{f}\left(\mathrm{g}_{1}\right)\right)\right), \mathrm{g}_{1}\right)$ and $G_{\mathrm{EXP}} \eta_{\mathrm{EXP}}=$ $p\left(g_{1}, f\left(g_{1}\right)\right) \vee r\left(g_{2}\left(f\left(g_{1}\right)\right)\right)$.

We now leave the consideration of how an interpolant lifting base can be constructed and view the interface provided by that concept from its other side, as a representation of the preconditions of interpolant lifting, that is, of the construction of a first-order CraigLyndon interpolant from the components of a given lifting base. This is made precise with the following theorem, which is proven later in the dedicated Section 9.

Theorem 21 (Interpolant Lifting) Let $F, G, \mathcal{F}, \mathcal{G}, H_{G R D}$ be the first components of a lifting base. Let $\mathcal{F G}$ stand for $\mathcal{F} \cup \mathcal{G}$. Let $\left\{t_{1}, \ldots, t_{n}\right\}$ be the set of the $\mathcal{F} \mathcal{G}$-terms with an $\mathcal{F G}$-termsmaximal occurrence in $H_{G R D}$, ordered such that if $t_{i} \triangleleft t_{j}$, then $i<j$. Let $\left\{v_{1}, \ldots, v_{n}\right\}$ be a set of fresh variables and let $\sigma$ be the injective substitution

$$
\sigma \stackrel{\text { def }}{=}\left\{v_{i} \mapsto t_{i} \mid i \in\{1, \ldots, n\}\right\} .
$$

For $i \in\{1, \ldots, n\}$ let $Q_{i} \stackrel{\text { def }}{=} \exists$ if $v_{i} \sigma \in \mathcal{F}$-terms and $Q_{i} \stackrel{\text { def }}{=} \forall$ otherwise, that is, if $v_{i} \sigma \in$ G-terms. Then

$$
Q_{1} v_{1} \ldots Q_{n} v_{n} H_{G R D}\left\langle\sigma^{-1}\right\rangle
$$

is a Craig-Lyndon interpolant of $F$ and $G$. 
Theorem 21 shows a construction of first-order formula from a given ground formula $H_{\text {GRD }}$ and two given sets, $\mathcal{F}$ and $\mathcal{G}$, of function symbols. The constructed formula is obtained from $H_{\text {GRD }}$ by replacing occurrences of $\mathcal{F}$-terms and $\mathcal{G}$-terms that are maximal with respect to $\mathcal{F} \cup \mathcal{G}$ with variables, and prepending a quantifier prefix upon these variables that respects certain constraints with respect to the replaced terms: Variables replacing an $\mathcal{F}$-term are existentially quantified, variables replacing a $\mathcal{G}$-term are universally quantified, and whenever variables $x, y$ replace terms $s, t$, respectively, such that $s \triangleleft t$, then the quantification upon $x$ precedes that upon $y$. The theorem then claims that the constructed first-order formula is a Craig-Lyndon interpolant of first-order formulas $F$ and $G$, provided $F, G, \mathcal{F}, \mathcal{G}, H_{\mathrm{GRD}}$ are the first components of a lifting base, that is, satisfy certain constraints that are expressed with reference to further related formulas $F_{\mathrm{GRD}}, F_{\mathrm{EXP}}$ and a related substitution $\eta_{\mathrm{EXP}}$. Interpolants obtained by applying the theorem are shown in the following example.

Example 22 (Interpolant Lifting) Consider the interpolant lifting bases from Example 20. Respective Craig-Lyndon interpolants obtained according to Theorem 21 are:

For (20.i) and (20.iv): $\forall v_{1} \exists v_{2} \mathrm{p}\left(v_{1}, v_{2}\right)$.

For (20.ii): $\exists v_{1} \forall v_{2} \exists v_{3} \mathrm{p}\left(v_{2}, v_{3}, v_{1}\right)$. Also other orderings of the quantifier prefix are possible according to Theorem 21. The only required condition is (expressed with the variable names of the shown value) that $\forall v_{2}$ must precede $\exists v_{3}$.

For (20.iii): $\exists v_{1} \forall v_{2} \forall v_{3}\left(\mathrm{p}\left(v_{2}, v_{1}\right) \wedge \mathrm{p}\left(v_{3}, v_{1}\right)\right)$, which is equivalent to $\exists v_{1} \forall v_{2} \mathrm{p}\left(v_{2}, v_{1}\right)$. Also arbitrary other orderings of the quantifier prefix are possible.

Note that for applying the lifting theorem only some of the components of a lifting base need actually to be constructed: $F, G, \mathcal{F}, \mathcal{G}$ and $H_{\mathrm{GRD}}$. For the remaining components, $F_{\mathrm{EXP}}$, $G_{\mathrm{EXP}}$ and $\eta_{\mathrm{EXP}}$, it is sufficient to ensure that they exist. They need not to be materialized. In other words, interpolant lifting according to the lifting theorem is applicable on top of all ground interpolation methods that produce from given $F, G, \mathcal{F}$ and $\mathcal{G}$ a ground formula $H_{\mathrm{GRD}}$ such that formulas $F_{\mathrm{EXP}}, G_{\mathrm{EXP}}$ and a substitutions $\eta_{\mathrm{EXP}}$ which satisfy the constraints for an interpolant lifting base exist, without need to actually compute them. Procedure 23 below gives an example of applying interpolant lifting on top of a ground interpolation method with clausal tableaux.

We conclude this section with a discussion on how the components of an interpolant lifting base $\left\langle F, G, \mathcal{F}, \mathcal{G}, H_{\mathrm{GRD}}, F_{\mathrm{EXP}}, G_{\mathrm{EXP}}, \eta_{\mathrm{EXP}}\right\rangle$ can be matched with the color-based terminology used in some of the literature on interpolation of refutations by resolution and superposition. We already noted aspects of that terminology in footnote 3 (page 8), and applied it in Definition 11. It has been developed mainly for clausal inputs, whereas our interpolation input formulas $F$ and $G$ are subject to Skolemization, which introduces fresh function symbols. As sketched in the proof of Proposition 19, Skolemization for interpolation can be performed separately on $F$ and $\neg G$. Interpolation for clausal inputs is considered as applied after such a separate Skolemization, implying that Skolem functions are colored.

Thus $\mathcal{F}$ may be taken as the set of F-colored symbols and $\mathcal{G}$ as the set of G-colored symbols, although both sets can contain Skolem functions that are present neither in $F$ nor in $G$. However, with respect to the quantifier-free formulas $F_{\mathrm{EXP}}$ and $G_{\mathrm{EXP}}$, the sets $\mathcal{F}$ and $\mathcal{G}$ are the sets of the F-and G-colored, respectively, symbols in the strict original sense.

A compound structure, that is, a term or a formula, is called F-colored if all symbols occurring in it are either F-colored or transparent and there is at least one occurrence of an F-colored symbol. The definition of G-colored for compound structures is analogous. Hence the formulas $F$ and $F_{\mathrm{EXP}}$ are either F-colored or transparent, and the formulas $G$ and $G_{\mathrm{EXP}}$ are either G-colored or transparent. Transparency of these formulas might be forbidden, as it indicates that inputs themselves already provide trivial interpolants. In our context this 
seems, however, an artificial restriction that is undesired because colored inputs $F$ and $G$ may lead to formulas $F_{\mathrm{EXP}}$ and $G_{\mathrm{EXP}}$ that are transparent, and, moreover, structural properties of interpolants for transparent inputs might be of interest to get insights on the interpolation algorithm and in the perspective of the computation of interpolants with further properties than those required of Craig-Lyndon interpolants.

Arbitrary colored and transparent function symbols from $F_{\mathrm{EXP}}$ and $G_{\mathrm{EXP}}$ are allowed in the formulas $H_{\mathrm{GRD}}, F_{\mathrm{EXP}} \eta_{\mathrm{EXP}}$ and $G_{\mathrm{EXP}} \eta_{\mathrm{EXP}}$, such that these formulas may have any of the four possible color status values (transparent, F-colored, G-colored, or FG-mixed). All predicates occurring in $H_{\mathrm{GRD}}$ are transparent. The key to interpolant lifting according to Theorem 21 is to consider occurrences of $\mathcal{F}$-terms and $\mathcal{G}$-terms that are maximal with respect to $\mathcal{F} \cup \mathcal{G}$. An $\mathcal{F}$-term is either F-colored or FG-mixed, a $\mathcal{G}$-term is either $\mathrm{G}$-colored or FG-mixed. However, an exact characterization of the $\mathcal{F}$-terms and $\mathcal{G}$-terms would require an extension of the color-based terminology that takes the color of the outermost symbol of a term especially into account.

\section{First-Order Interpolation with Clausal Tableaux}

Based on the correctness of ground interpolant extraction with the ipol function (Lemma 7) and the interpolant lifting theorem (Theorem 21) we can now formulate a generalization of the CTI procedure (Procedure 8) that computes Craig-Lyndon interpolants from first-order sentences (without equality, except if represented as predicate, see Sect. 10.4). We call the procedure CTIF, suggesting Clausal Tableau Interpolation for First-Order Formulas.

\section{Procedure 23 (The CTIF Method for Craig-Lyndon Interpolation)}

INPUT: First-order sentences $F$ and $G$ such that $F \models G$.

Method:

1. Skolemization and clausification: Apply conversion to prenex form and Skolemization to $F$ and to $\neg G$, independently to each formula, to obtain disjoint sets of fresh Skolem functions $\mathcal{F}^{\prime}, \mathcal{G}^{\prime}$, clausal formulas $F^{\prime}, G^{\prime}$, and sets $\mathcal{U}^{\prime}=\operatorname{Var}\left(F^{\prime}\right), \mathcal{V}^{\prime}=\operatorname{Var}\left(G^{\prime}\right)$ of variables such that:

$$
\begin{aligned}
& \text { (a) } F \equiv \exists \mathcal{F}^{\prime} \forall \mathcal{U}^{\prime} F^{\prime} \text { and } \neg G \equiv \exists \mathcal{G}^{\prime} \forall \mathcal{V}^{\prime} G^{\prime} \text {. } \\
& \text { (b) } \mathcal{V} o c^{ \pm}\left(F^{\prime}\right) \subseteq \mathcal{V} o c^{ \pm}(F) \cup \mathcal{F}^{\prime} \text { and } \mathcal{V} o c^{ \pm}\left(\neg G^{\prime}\right) \subseteq \mathcal{V} o c^{ \pm}(G) \cup \mathcal{G}^{\prime} \text {. } \\
& \text { (c) } \forall \mathcal{U}^{\prime} \forall \mathcal{V}^{\prime}\left(F^{\prime} \wedge G^{\prime}\right) \vDash \perp \text {. }
\end{aligned}
$$

2. Tableau computation: Compute a closed clausal tableau for the clausal formula $F^{\prime} \wedge G^{\prime}$. If the tableau is not already leaf-closed, convert it to leaf-closed form by removing all edges that originate in closing nodes.

3. Tableau grounding: (Recall that the tableau may have rigid variables, that is, variables whose scope is the whole tableau may occur in literal labels.) Instantiate all variables of the tableau with arbitrary ground terms constructed from symbols in $\mathcal{F} u n\left(F^{\prime} \wedge G^{\prime}\right)$ and, if there is no constant in this set of function symbols, a fresh constant $\mathrm{c}_{0}$. (Options for choosing these ground terms will be discussed in Sect. 10.2.) Observe that the grounded tableau is still a leaf-closed tableau for $F^{\prime} \wedge G^{\prime}$.

4. Side assignment: Convert the ground tableau to a two-sided tableau for $F^{\prime}$ and $G^{\prime}$ by attaching appropriate side labels to all nodes except the root. This is always possible because every clause of the tableau is an instance of a clause in $F^{\prime}$ or in $G^{\prime}$. (It is possible that a clause of the tableau is an instance of a clause in $F^{\prime}$ and of a clause in $G^{\prime}$. See Sect. 10.2.) 
5. Ground interpolant extraction: Let $H_{\mathrm{GRD}}$ be the value of ipol $\left(N_{0}\right)$, where $N_{0}$ is the root of the tableau.

6. Interpolant lifting: Let $\mathcal{F} \stackrel{\text { def }}{=} \mathcal{F}^{\prime} \cup(\mathcal{F} u n(F) \backslash \mathcal{F} u n(G)) \cup\left\{\mathrm{c}_{0}\right\}$ and let $\mathcal{G} \stackrel{\text { def }}{=} \mathcal{G}^{\prime} \cup(\mathcal{F} u n(G) \backslash$ $\mathcal{F} u n(F))$. ( $\mathrm{c}_{0}$ needs only to be considered if it has been introduced in step 3 because there is no constant occurring in the clausal formula $F^{\prime} \wedge G^{\prime}$. It may here be placed also in $\mathcal{G}$ instead of $\mathcal{F}$.) Observe that $F, G, \mathcal{F}, \mathcal{G}, H_{\text {GRD }}$ form the first components of an interpolant lifting base. Let $H$ be the Craig-Lyndon interpolant of $F$ and $G$ specified in Theorem 21 with respect to $F, G, \mathcal{F}, \mathcal{G}, H_{\mathrm{GRD}}$ as first components of an interpolant lifting base.

OutPuT: Return $H$. The output is a Craig-Lyndon interpolant of the input sentences.

Skolemization and clausification (step 1) might integrate preprocessing operations such as structural normal form conversion and versions of well-known clausal preprocessing techniques that, however, need to be specially adapted as for interpolation it is not sufficient to just preserve unsatisfiability. This is discussed below in Sect. 10.3. The tableau computation itself (step 2) is just a refutation task, entirely independent from its use for interpolation. That is, a clausal tableau prover can be used there without making any changes to its internal workings. The duplication and instantiation of the input first-order clauses is in essence performed in this step by the tableau prover. ${ }^{13}$ Nevertheless, tableau construction methods may yield tableaux in which variables are instantiated through unification just as far as required to ensure that the tableau is closed. The purpose of the tableau grounding (step 3) is to instantiate any remaining variables in the closed tableau to ground terms. This is a mere linear operation, where, however, different options are possible that have effect on the resulting interpolant as discussed in Sect. 10.2. Side assignment (step 4) and ground interpolant extraction (step 5) then operate on the resulting ground tableau.

A lifting base $\left\langle F, G, \mathcal{F}, \mathcal{G}, H_{\mathrm{GRD}}, F_{\mathrm{EXP}}, G_{\mathrm{EXP}}, \eta_{\mathrm{EXP}}\right\rangle$ that justifies the application of interpolant lifting (step 6) can be determined as follows: Let $F, G, \mathcal{F}, \mathcal{G}, H_{\mathrm{GRD}}$ are as specified in the procedure description. To determine the remaining components consider the tableau after grounding and side assignment. Take as $F_{\mathrm{EXP}} \eta_{\mathrm{EXP}}$ the conjunction of the tableau clauses with side $\mathrm{F}$ and as $G_{\mathrm{EXP}} \eta_{\mathrm{EXP}}$ the negation of the conjunction of the tableau clauses with side G. By comparison with the clausal formulas $F^{\prime}$ and $G^{\prime}$ constructed by the procedure, the formulas $F_{\mathrm{EXP}} \eta_{\mathrm{EXP}}$ and $G_{\mathrm{EXP}} \eta_{\mathrm{EXP}}$ can be separated into suitable formulas $F_{\mathrm{EXP}}$ and $G_{\mathrm{EXP}}$ and a substitution $\eta_{\text {EXP }}$.

The procedure can easily be adapted to handle not just sentences, but also formulas with free variables as in- and outputs, as required by the full definition of Craig-Lyndon interpolant: In a preprocessing step, the free variables of $F$ and $G$ would be converted to constants and in a postprocessing step the occurrences in $H$ would be converted back to the corresponding free variables.

If the invoked method for tableau computation is complete, that is, it computes a closed clausal tableau for all unsatisfiable inputs, then, like the CTI procedure, the CTIF procedure is complete, that is, it outputs a Craig-Lyndon interpolant of its inputs $F$ and $G$ whenever $F \vDash G$. Also as for CTI, the size of the result formula is linear in the size of the clausal tableau, or more precisely in the number of its leaves whose target has the opposite side label. The lifting step does not change this. (Of course, as for CTI, the size of the tableau itself is not polynomially bounded.)

13 This contrast to approaches like [21], where instantiation is performed specifically for interpolation. 


\section{Proof of the Interpolant Lifting Theorem}

In this section we prove Theorem 21 . The proof statement is given at the end of the section. It resides on the definition of interpolant lifting base (Definition 16) and a lemma concerning the semantic properties of interpolants that is developed throughout the section. We start from a given interpolant lifting base. Further auxiliary formulas, quantifiers, variables and substitutions are then defined and propositions that relate them are stated. These auxiliary elements are specified in definition environments, which are used here differently from the other sections to specify elements that are only of relevance within the section. Correspondingly, the definitions and statements in this section directly refer to the components from the given interpolant lifting base and elements defined previously in the section.

To prove Theorem 21, it has to be shown that the sentence $H=Q_{1} v_{1} \ldots Q_{n} v_{n} H_{\mathrm{GRD}}\left\langle\sigma^{-1}\right\rangle$, as specified in the theorem is a Craig-Lyndon interpolant of sentences $F$ and $G$. This is the case if $F \mid=H, H \vDash G$ and $\mathcal{V}_{o c}^{ \pm}(H) \subseteq \mathcal{V}_{o c}^{ \pm}(F) \cap \mathcal{V}^{ \pm} c^{ \pm}(G)$. Of the two semantic conditions, we focus on $F \mid=H$, as $H \models G$ can be shown analogously. We assume in this section a context with a given interpolant lifting base

$$
\left\langle F, G, \mathcal{F}, \mathcal{G}, H_{\mathrm{GRD}}, F_{\mathrm{EXP}}, G_{\mathrm{EXP}}, \eta_{\mathrm{EXP}}\right\rangle
$$

and the symbols

$$
\mathcal{F G}, \sigma, v_{1}, \ldots, v_{n}, \text { and } Q_{1}, \ldots, Q_{n}
$$

defined as in the preconditions of Theorem 21 . The following definition specifies, along with some shorthands, an injective ground substitution $\eta_{\mathrm{LFT}}$ that extends the lifting substitution $\sigma$ by ranging over all $\mathcal{F} \mathcal{G}$-terms that occur in $F_{\mathrm{EXP}} \eta_{\mathrm{EXP}}$ or in $H_{\mathrm{GRD}}$.

Definition 24 (Formula $F_{\mathrm{GRD}}$, Quantified Variables $Q_{1}^{\prime} v_{1}^{\prime}, \ldots, Q_{m}^{\prime} v_{m}^{\prime}$, Injective Substitution $\eta_{\mathrm{LFT}}$, Sets of Variables $\mathcal{X}, \mathcal{Y}$, Formula $F_{\mathrm{LFT}}$ )

(i) Define the ground formula

$$
F_{\mathrm{GRD}} \stackrel{\text { def }}{=} F_{\mathrm{EXP}} \eta_{\mathrm{EXP}} \text {. }
$$

(ii) Define a set of variables $\left\{v_{1}^{\prime}, \ldots, v_{m}^{\prime}\right\} \supseteq\left\{v_{1}, \ldots, v_{n}\right\}$ and an injective substitution $\eta_{\text {LFT }}$ (the subscript LFT suggesting lifting) with the following properties:

(a) $\operatorname{Dom}\left(\eta_{\mathrm{LFT}}\right)=\left\{v_{1}^{\prime}, \ldots, v_{m}^{\prime}\right\}$.

(b) $\operatorname{Rng}\left(\eta_{\mathrm{LFT}}\right)=\left\{t \mid t\right.$ is an $\mathcal{F G}$-term occurring in $F_{\mathrm{GRD}}$ or in $\left.H_{\mathrm{GRD}}\right\}$.

(c) If $v_{i}^{\prime} \eta_{\mathrm{LFT}} \triangleleft v_{j}^{\prime} \eta_{\mathrm{LFT}}$, then $i<j$.

(d) $\left\{v_{1}, \ldots, v_{n}\right\} \subseteq\left\{v_{1}^{\prime}, \ldots, v_{m}^{\prime}\right\}$.

(e) If $v_{i}^{\prime}=v_{j}$, then $v_{i}^{\prime} \eta_{\mathrm{LFT}}=v_{j} \eta_{\mathrm{LFT}}$.

(f) If $v_{i}^{\prime}=v_{j}, v_{k}^{\prime}=v_{l}$ and $j<l$, then $i<k$.

(iii) Define the shorthands $\mathcal{X} \stackrel{\text { def }}{=}\left\{v_{i}^{\prime} \mid v_{i}^{\prime} \eta_{\mathrm{LFT}} \in \mathcal{F}\right.$-terms $\}$ and $\mathcal{Y} \stackrel{\text { def }}{=}\left\{v_{i}^{\prime} \mid v_{i}^{\prime} \eta_{\mathrm{LFT}} \in \mathcal{G}\right.$-terms $\}$.

(iv) For $i \in\{1, \ldots, m\}$ define $Q_{i}^{\prime} \stackrel{\text { def }}{=} \forall$ if $v_{i}^{\prime} \in \mathcal{Y}$ and define $Q_{i}^{\prime} \stackrel{\text { def }}{=} \exists$ otherwise, that is, if $v_{i}^{\prime} \in \mathcal{X}$.

(v) Define the quantifier-free formula

$$
F_{\mathrm{LFT}} \stackrel{\text { def }}{=} F_{\mathrm{GRD}}\left\langle\eta_{\mathrm{LFT}}^{-1}\right\rangle .
$$

Definition 24.ii characterizes the specified elements just in terms of properties. The following proposition supplements this by claiming their existence and supplementing a construction in its proof. 
Proposition 25 (Existence of $\left\{\boldsymbol{v}_{\mathbf{1}}^{\prime}, \ldots, v_{\boldsymbol{m}}^{\prime}\right\}$ and $\boldsymbol{\eta}_{\mathrm{LFT}}$ ) There exists a set $\left\{v_{1}^{\prime}, \ldots, v_{m}^{\prime}\right\}$ of variables and a substitution $\eta_{L F T}$ as specified in Definition 24.ii.

Proof The set $\left\{v_{1}^{\prime}, \ldots, v_{m}^{\prime}\right\}$ of variables and the substitution $\eta_{\mathrm{LFT}}$ can be constructed as follows: Collect all $\mathcal{F} G$-terms occurring in $F_{\mathrm{GRD}}$ or $H_{\mathrm{GRD}}$. They form a finite set of ground terms, a superset of $\left\{v_{1} \sigma, \ldots, v_{n} \sigma\right\}$. Let $\left\{t_{1}, \ldots, t_{m}\right\}$ be this set, ordered such that $t_{1}<$ $\ldots<t_{m}$ extends the ordering $v_{1} \sigma<\ldots<v_{n} \sigma$ (i.e., if $t_{i}=v_{k} \sigma, t_{j}=v_{l} \sigma$ and $k<l$, then $i<j$ ) and that if $t_{i} \triangleleft t_{j}$, then $i<j$. Define $\eta_{\mathrm{LFT}} \stackrel{\text { def }}{=}\left\{v_{1}^{\prime} \mapsto t_{1}, \ldots, v_{m}^{\prime} \mapsto t_{m}\right\}$.

The injective substitution $\eta_{\mathrm{LFT}}$ extends $\sigma$ that was specified in the preconditions of the interpolant lifting theorem with pairs that map additional variables to $\mathcal{F} \mathcal{G}$-terms that have an occurrence in $F_{\mathrm{GRD}}$ or $H_{\mathrm{GRD}}$ which is not maximal or is not in $H_{\mathrm{GRD}}$. Correspondingly, the quantifier prefix $Q_{1}^{\prime} v_{1}^{\prime} \ldots Q_{m}^{\prime} v_{m}^{\prime}$ "includes" $Q_{1} v_{1} \ldots Q_{n} v_{n}$. That is, $Q_{1}^{\prime} v_{1}^{\prime} \ldots Q_{m}^{\prime} v_{m}^{\prime}$ could be obtained from $Q_{1} v_{1} \ldots Q_{n} v_{n}$ by adding to the front, the end, or in-between additional quantifications upon those variables in $\left\{v_{1}^{\prime}, \ldots, v_{m}^{\prime}\right\}$ that are not in $\left\{v_{1}, \ldots, v_{n}\right\}$. We now define the shorthand $H_{\mathrm{LFT}}$ for the quantifier-free formula that follows the quantifier prefix in the interpolant lifting result.

Definition 26 (Formula $\boldsymbol{H}_{\text {LFT }}$ ) Define the quantifier-free formula

$$
H_{\mathrm{LFT}} \stackrel{\text { def }}{=} H_{\mathrm{GRD}}\left\langle\eta_{\mathrm{LFT}}^{-1}\right\rangle
$$

We note the following properties of $H_{\mathrm{LFT}}$.

\section{Proposition 27 (Properties of $\boldsymbol{H}_{\mathrm{LFT}}$ )}

(i) $H_{L F T}=H_{G R D}\left\langle\sigma^{-1}\right\rangle$.

(ii) $F_{L F T} \vDash H_{L F T}$.

(iii) $Q_{1}^{\prime} v_{1}^{\prime} \ldots Q_{m}^{\prime} v_{m}^{\prime} H_{L F T} \equiv Q_{1} v_{1} \ldots Q_{n} v_{n} H_{L F T}$.

Proof (27.i) By the definitions of $\eta_{\mathrm{LFT}}$ and $\sigma$ the inverse application of either substitution to $H_{\text {GRD }}$ has the effect that all $\mathcal{F} \mathcal{G}$-terms-maximal occurrences of $\mathcal{F} \mathcal{G}$-terms are replaced with variables, and, moreover, ensured by conditions (d) and (e) of Definition 24.ii, in both cases the same variables. (27.ii) By condition (vii) of the characterization of lifting base, Definition $16, H_{\mathrm{GRD}}$ is a Craig-Lyndon interpolant of $F_{\mathrm{EXP}} \eta_{\mathrm{EXP}}$. Hence $F_{\mathrm{EXP}} \eta_{\mathrm{EXP}}=H_{\mathrm{GRD}}$, which, by the definition of $F_{\mathrm{GRD}}$ can be expressed as $F_{\mathrm{GRD}}=H_{\mathrm{GRD}}$. By the definitions of $F_{\mathrm{LFT}}$ and $H_{\mathrm{LFT}}$ the proposition statement can be expressed as $F_{\mathrm{GRD}}\left\langle\eta_{\mathrm{LFT}}^{-1}\right\rangle \vDash H_{\mathrm{GRD}}\left\langle\eta_{\mathrm{LFT}}^{-1}\right\rangle$, which follows from $F_{\mathrm{GRD}} \vDash H_{\mathrm{GRD}}$ by Proposition 15.ii. (27.iii) Follows from the definitions of the prefixes $Q_{1}^{\prime} v_{1}^{\prime} \ldots Q_{m}^{\prime} v_{m}^{\prime}$ and $Q_{1} v_{1} \ldots Q_{n} v_{n}$ since $\operatorname{Var}\left(H_{\mathrm{LFT}}\right) \subseteq\left\{v_{1}, \ldots, v_{n}\right\} \subseteq\left\{v_{1}^{\prime}, \ldots, v_{m}^{\prime}\right\}$.

The following example illustrate the elements introduced so far.

Example 28 (Formulas, Substitutions and Quantifier Prefixes Introduced So Far) The following table shows values for formulas, substitutions and quantifier prefixes defined so far in this section for the lifting base from Example 20.iv as starting point. Properties stated 
with Proposition 27 can be easily verified for the example values.

$$
\begin{aligned}
F & =\forall x \mathrm{p}(x, \mathrm{f}(x)) \wedge \forall x \forall y \mathrm{q}(\mathrm{f}(x), y) . \\
\mathcal{F} & =\{\mathrm{f}\} . \\
\mathcal{G} & =\left\{\mathrm{g}_{1}, \mathrm{~g}_{2}\right\} . \\
H_{\mathrm{GRD}} & =\mathrm{p}\left(\mathrm{g}_{1}, \mathrm{f}\left(\mathrm{g}_{1}\right)\right) . \\
\sigma & =\left\{v_{1} \mapsto \mathrm{g}_{1}, v_{2} \mapsto \mathrm{f}\left(\mathrm{g}_{1}\right)\right\} . \\
Q_{1} v_{1} \ldots Q_{m} v_{m} & =\forall v_{1} \exists v_{2} . \\
H & =\forall v_{1} \exists v_{2} \mathrm{p}\left(v_{1}, v_{2}\right) . \\
F_{\mathrm{EXP}} & =\mathrm{p}\left(u_{1}, \mathrm{f}\left(u_{1}\right)\right) \wedge \mathrm{q}\left(\mathrm{f}\left(u_{3}\right), u_{4}\right) . \\
\eta_{\mathrm{EXP}} & =\left\{u_{1} \mapsto \mathrm{g}_{1}, u_{2} \mapsto \mathrm{f}\left(\mathrm{g}_{1}\right), u_{3} \mapsto \mathrm{g}_{2}\left(\mathrm{f}\left(\mathrm{g}_{1}\right)\right), u_{4} \mapsto \mathrm{g}_{1}\right\} . \\
F_{\mathrm{GRD}}=F_{\mathrm{EXP}} \eta_{\mathrm{EXP}} & =\mathrm{p}\left(\mathrm{g}_{1}, \mathrm{f}\left(\mathrm{g}_{1}\right)\right) \wedge \mathrm{q}\left(\mathrm{f}\left(\mathrm{g}_{2}\left(\mathrm{f}\left(\mathrm{g}_{1}\right)\right)\right), \mathrm{g}_{1}\right) . \\
\eta_{\mathrm{LFT}} & \left.=\left\{v_{1}^{\prime} \mapsto \mathrm{g}_{1}, v_{2}^{\prime} \mapsto \mathrm{f}\left(\mathrm{g}_{1}\right), v_{3}^{\prime} \mapsto \mathrm{g}_{2}\left(\mathrm{f}\left(\mathrm{g}_{1}\right)\right), v_{4}^{\prime} \mapsto \mathrm{f}\left(\mathrm{g}_{2}\left(\mathrm{f}\left(\mathrm{g}_{1}\right)\right)\right)\right)\right\}, \\
\mathcal{X} & =\left\{v_{2}^{\prime}, v_{4}^{\prime}\right\} . \\
\mathcal{Y} & =\left\{v_{1}^{\prime}, v_{3}^{\prime}\right\} . \\
Q_{1}^{\prime} v_{1}^{\prime} \ldots Q_{1}^{\prime} v_{m}^{\prime} & =\forall v_{1}^{\prime} \exists v_{2}^{\prime} \forall v_{3}^{\prime} \exists v_{4}^{\prime} . \\
F_{\mathrm{LFT}}=F_{\mathrm{GRD}}\left\langle\eta_{\mathrm{LFT}}^{-1}\right\rangle & =\mathrm{p}\left(v_{1}^{\prime}, v_{2}^{\prime}\right) \wedge \mathrm{q}\left(v_{4}^{\prime}, v_{1}^{\prime}\right) . \\
H_{\mathrm{LFT}}=H_{\mathrm{GRD}}\left\langle\eta_{\mathrm{LFT}}^{-1}\right\rangle & =\mathrm{p}\left(v_{1}^{\prime}, v_{2}^{\prime}\right) .
\end{aligned}
$$

Based on variable sets $\mathcal{X}$ and $\mathcal{Y}$ we now define a series of substitutions and a series of subsets of variables that will be used later in an induction.

Definition 29 (Substitutions $\boldsymbol{\phi}_{i}$ and Variable Sets $\boldsymbol{y}_{i}$ ) For $i \in\{0, \ldots, m\}$ define substitutions $\phi_{i}$ and sets $\mathcal{Y}_{i}$ of variables as follows:

(i) $\phi_{i} \stackrel{\text { def }}{=}\left\{v_{j}^{\prime} \mapsto v_{j}^{\prime} \eta_{\mathrm{LFT}}\left\langle\left.\eta_{\mathrm{LFT}}\right|_{y} ^{-1}\right\rangle \mid v_{j}^{\prime} \in \mathcal{X}\right.$ and $\left.j>i\right\}$.

(ii) $\mathcal{Y}_{i} \stackrel{\text { def }}{=}\left\{v_{j}^{\prime} \mid v_{j}^{\prime} \in \mathcal{Y}\right.$ and $\left.j>i\right\}$.

(iii) In addition, define the shorthand $\phi_{\mathrm{LFT}} \stackrel{\text { def }}{=} \phi_{0}$. (Observe that $\mathcal{Y}=\mathcal{Y}_{0}$.)

The domain of substitution $\phi_{i}$ is the set of those $v_{j}^{\prime}$ with $j$ strictly larger than $i$ that are mapped by $\eta_{\mathrm{LFT}}$ to an $\mathcal{F}$-term. The value of $v_{j}^{\prime} \phi_{i}$ is the value of $v_{j}^{\prime} \eta_{\mathrm{LFT}}($ an $\mathcal{F}$-term) after replacing all maximal occurrences of $\mathcal{G}$-terms with the respective variables $v_{k}^{\prime}$ that are mapped by $\eta_{\mathrm{LFT}}$ to them. For Example 28, we obtain

$$
\begin{aligned}
\phi_{\mathrm{LFT}}=\phi_{0}=\phi_{1} & =\left\{v_{2}^{\prime} \mapsto \mathrm{f}\left(v_{1}^{\prime}\right), v_{4}^{\prime} \mapsto \mathrm{f}\left(v_{3}^{\prime}\right)\right\} . \\
\phi_{2}=\phi_{3} & =\left\{v_{4}^{\prime} \mapsto \mathrm{f}\left(v_{3}^{\prime}\right)\right\} . \\
\phi_{4}=\phi_{m} & =\varepsilon .
\end{aligned}
$$

The set of variables $\mathcal{Y}_{i}$ is the sets of those $v_{j}^{\prime}$ with $j$ strictly larger than $i$ that are mapped by $\eta_{\text {LFT }}$ to a $\mathcal{G}$-term. For Example 28 the values of $\mathcal{Y}_{i}$ are $\mathcal{Y}=\mathcal{Y}_{0}=\left\{v_{1}^{\prime}, v_{3}^{\prime}\right\}, \mathcal{Y}_{1}=\mathcal{Y}_{2}=\left\{v_{3}^{\prime}\right\}$, and $\mathcal{Y}_{3}=\mathcal{Y}_{4}=\mathcal{Y}_{m}=\emptyset$. Members of $\mathcal{X}$ have the following properties, which will be used later in the proof of Lemma 34.

Proposition 30 (Properties of Members of $\mathcal{X}$ ) For all $v_{i}^{\prime} \in \mathcal{X}$ it holds that

(i) $v_{i}^{\prime} \notin \mathcal{V} \mathcal{R} n g\left(\phi_{i}\right)$.

(ii) $\mathcal{Y}_{i} \cap \operatorname{Var}\left(v_{i}^{\prime} \phi_{i-1}\right)=\emptyset$.

Proof (30.i) From the definitions of $\phi_{i}$ and $\mathcal{Y}$ and given that $\eta_{\mathrm{LFT}}$ is a ground substitution it follows that $\mathcal{V} \operatorname{Rng}\left(\phi_{i}\right) \subseteq \mathcal{Y}$. Since $\mathcal{X} \cap \mathcal{Y}=\emptyset$, the proposition then follows from the precondition $v_{i}^{\prime} \in \mathcal{X}$. 
(30.ii) We derive a contradiction from assuming that, contrary to the proposition, there exists a $j$ such that:

(1) $v_{j}^{\prime} \in \mathcal{Y}_{i}$.

(2) $v_{j}^{\prime} \in \mathcal{V} \operatorname{Var}\left(v_{i}^{\prime} \phi_{i-1}\right)$.

The derivation proceeds in the following steps explained below:

(3) $j>i$.

(4) $\operatorname{Var}\left(v_{i}^{\prime} \eta_{\mathrm{LFT}}\right)=\emptyset$.

(5) $v_{i}^{\prime} \eta_{\mathrm{LFT}}=v_{i}^{\prime} \eta_{\mathrm{LFT}}\left\langle\left.\eta_{\mathrm{LFT}}\right|_{y} ^{-1}\right\rangle \eta_{\mathrm{LFT}} \mid y$.

(6) $v_{i}^{\prime} \eta_{\mathrm{LFT}}=v_{i}^{\prime} \eta_{\mathrm{LFT}}\left\langle\left.\eta_{\mathrm{LFT}}^{-1}\right|_{y} ^{-1}\right\rangle \eta_{\mathrm{LFT}}$.

(7) $v_{i}^{\prime} \phi_{i-1}=v_{i}^{\prime} \eta_{\mathrm{LFT}}\left\langle\eta_{\mathrm{LFT}}\right| \begin{aligned} & -1 \\ & y\end{aligned}$.

(8) $v_{i}^{\prime} \eta_{\mathrm{LFT}}=v_{i}^{\prime} \phi_{i-1} \eta_{\mathrm{LFT}}$.

(9) $v_{j}^{\prime} \eta_{\mathrm{LFT}} \triangleleft v_{i}^{\prime} \phi_{i-1} \eta_{\mathrm{LFT}}$.

(10) $v_{j}^{\prime} \eta_{\mathrm{LFT}} \triangleleft v_{i}^{\prime} \eta_{\mathrm{LFT}}$.

(11) $j<i$.

Step (3) follows from (1) and the definition of $\boldsymbol{y}_{i}$. Step (4) holds since $\eta_{\text {LfT }}$ is a ground substitution and $v_{i}^{\prime} \in \operatorname{Dom}\left(\eta_{\mathrm{LFT}}\right)$. Step (5) follows from Proposition 15.i, whose precondition $\operatorname{Dom}\left(\eta_{\mathrm{LFT}} \mid y\right) \cap \mathcal{V} \operatorname{Var}\left(v_{i}^{\prime} \eta_{\mathrm{LFT}}\right)=\emptyset$ is implied by (4). Step (6) follows from (5) and (4); step (7) from the definition of $\phi_{i-1}$; step (8) from (6) and (7); step (9) from (2) and the definition of $\phi_{i-1}$, which precludes that $v_{i}^{\prime} \phi_{i-1}$ is just a variable; step (10) from (9) and (8); and, finally, step (11), which contradicts (3), from (10) and property (c) of Definition 24.ii.

Both formulas $F_{\mathrm{EXP}}$ and $F_{\mathrm{LFT}}$ generalize the ground formula $F_{\mathrm{GRD}}$, but in different ways, which are reconciled by observing that $F_{\mathrm{LFT}} \phi_{\mathrm{LFT}}$ is a (possibly non-ground) instance of $F_{\text {EXP. }}$. As an example consider $F_{\mathrm{GRD}}=\mathrm{p}(\mathrm{f}(\mathrm{g}(\mathrm{f}(\mathrm{a}))), \mathrm{g}(\mathrm{f}(\mathrm{a})), \mathrm{g}(\mathrm{f}(\mathrm{a})))$, which is an instance of both $F_{\mathrm{EXP}}=\mathrm{p}\left(\mathrm{f}\left(u_{1}\right), u_{1}, u_{2}\right)$ and $F_{\mathrm{LFT}}=\mathrm{p}(x, y, y)$. If $\phi_{\mathrm{LFT}}=\{x \mapsto \mathrm{f}(y)\}$, then $F_{\mathrm{LFT}} \phi_{\mathrm{LFT}}=$ $\mathrm{p}(\mathrm{f}(y), y, y)$ is more general than $F_{\mathrm{GRD}}$ and an instance of both $F_{\mathrm{EXP}}$ and $F_{\mathrm{LFT}}$. (This example will be fleshed out further in Example 33 below.) The following definition specifies the substitution $\phi_{\mathrm{EXP}}$, which is then used in Proposition 32 to justify that $F_{\mathrm{LFT}} \phi_{\mathrm{LFT}}$ is an instance of $F_{\mathrm{EXP}}$.

Definition 31 (Substitution $\phi_{\mathbf{E X P}}$ ) Define the substitution $\phi_{\mathrm{EXP}}$ as

$$
\phi_{\mathrm{EXP}} \stackrel{\text { def }}{=}\left\{u \mapsto u \eta_{\mathrm{EXP}}\left\langle\left.\eta_{\mathrm{LFT}}\right|_{y} ^{-1}\right\rangle \mid u \in \operatorname{Var}\left(F_{\mathrm{EXP}}\right)\right\} .
$$

\section{Proposition 32 (Key Property of $\phi_{\mathrm{EXP}}$ )}

$$
F_{E X P} \phi_{E X P}=F_{L F T} \phi_{L F T} .
$$

Proof We show this with the help of two auxiliary substitutions $\theta_{\mathrm{EXP}}$ and $\theta_{\mathrm{LFT}}$ and an auxiliary quantifier-free formula $F_{\mathrm{TOP}}$ that generalizes $F_{\mathrm{EXP}}$ and $F_{\mathrm{LFT}}$. These auxiliary objects are not used elsewhere in the proof of Theorem 21. Let $\left\{t_{1}, \ldots, t_{k}\right\}$ be the set of all $\mathcal{F}$-terms with an $\mathcal{F}$-terms-maximal occurrence in $F_{\text {EXP }}$. Let $u_{1}, \ldots, u_{k}$ be fresh variables, define the substitution $\theta_{\text {EXP }}$ as

$$
\theta_{\mathrm{EXP}} \stackrel{\text { def }}{=}\left\{u_{i} \mapsto t_{i} \mid i \in\{1, \ldots, k\}\right\},
$$

and define the quantifier-free formula $F_{\mathrm{TOP}}$ as

$$
F_{\text {TOP }} \stackrel{\text { def }}{=} F_{\mathrm{EXP}}\left\langle\theta_{\mathrm{EXP}}^{-1}\right\rangle .
$$


Since $\operatorname{Dom}\left(\theta_{\mathrm{EXP}}\right) \cap \mathcal{V} \operatorname{Var}\left(F_{\mathrm{EXP}}\right)=\emptyset$ it follows from Proposition 15.i that $F_{\mathrm{EXP}}\left\langle\theta_{\mathrm{EXP}}^{-1}\right\rangle \theta_{\mathrm{EXP}}=F_{\mathrm{EXP}}$. Thus:

(1) $F_{\mathrm{TOP}} \theta_{\mathrm{EXP}}=F_{\mathrm{EXP}}$.

Define the substitution $\theta_{\mathrm{LFT}}$ as

$$
\theta_{\mathrm{LFT}} \stackrel{\text { def }}{=}\left\{u \mapsto u \theta_{\mathrm{EXP}} \eta_{\mathrm{EXP}}\left\langle\eta_{\mathrm{LFT}}^{-1}\right\rangle \mid u \in \operatorname{Var}\left(F_{\mathrm{TOP}}\right)\right\} .
$$

Because the range of $\eta_{\mathrm{LFT}}$ contains only $\mathcal{F G}$-terms and in $F_{\text {TOP }}$ there are no occurrences of $\mathcal{F G}$-terms, inversely applying $\eta_{\mathrm{LFT}}$ to an instance of $F_{\mathrm{TOP}}$, say $F_{\mathrm{TOP}} \xi$, yields the same result as applying to $F_{\text {TоP }}$ the substitution that maps each variable $u$ occurring in $F_{\text {TоР }}$ to the value of $u \xi$ after inversely applying $\eta_{\mathrm{LFT}}$. For the particular case of $\theta_{\mathrm{EXP}} \eta_{\mathrm{EXP}}$ in the role of $\xi$ this can be stated formally as

(2) $F_{\mathrm{TOP}} \theta_{\mathrm{EXP}} \eta_{\mathrm{EXP}}\left\langle\eta_{\mathrm{LFT}}^{-1}\right\rangle=F_{\mathrm{TOP}}\left\{u \mapsto u \theta_{\mathrm{EXP}} \eta_{\mathrm{EXP}}\left\langle\eta_{\mathrm{LFT}}^{-1}\right\rangle \mid u \in \operatorname{Var}\left(F_{\mathrm{TOP}}\right)\right\}$.

Observe that the right side of (2) is equal to $F_{\mathrm{TOP}} \theta_{\mathrm{LFT}}$. By (1) and by taking into account the definitions of $F_{\mathrm{GRD}}$ and $F_{\mathrm{LFT}}$ we can conclude via $F_{\mathrm{TOP}} \theta_{\mathrm{LFT}}=F_{\mathrm{TOP}} \theta_{\mathrm{EXP}} \eta_{\mathrm{EXP}}\left\langle\eta_{\mathrm{LFT}}^{-1}\right\rangle=$ $F_{\mathrm{EXP}} \eta_{\mathrm{EXP}}\left\langle\eta_{\mathrm{LFT}}^{-1}\right\rangle=F_{\mathrm{GRD}}\left\langle\eta_{\mathrm{LFT}}^{-1}\right\rangle=F_{\mathrm{LFT}}$ that

(3) $F_{\mathrm{TOP}} \theta_{\mathrm{LFT}}=F_{\mathrm{LFT}}$.

Recall that $F_{\mathrm{GRD}}$ is a ground formula and that $\eta_{\mathrm{LFT}}$ is an injective substitution whose range includes all $\mathcal{F} \mathcal{G}$-terms occurring in $F_{\mathrm{GRD}}$. Inversely applying the restriction of $\eta_{\mathrm{LFT}}$ to $\mathcal{G}$-terms to $F_{\mathrm{GRD}}$ has the same result as inversely applying $\eta_{\mathrm{LFT}}$ to $F_{\mathrm{GRD}}$ followed by replacing those variables that are mapped by $\eta_{\mathrm{LFT}}$ to an $\mathcal{F}$-term with the result of inversely applying to that respective $\mathcal{F}$-term the restriction of $\eta_{\mathrm{LFT}}$ to $\mathcal{G}$-terms. With the first description on the right side, this equality is formally stated as

(4) $F_{\mathrm{GRD}}\left\langle\eta_{\mathrm{LFT}}^{-1}\right\rangle\left\{x \mapsto x \eta_{\mathrm{LFT}}\left\langle\left.\eta_{\mathrm{LFT}}\right|_{y} ^{-1}\right\rangle \mid x \in \mathcal{X}\right\}=F_{\mathrm{GRD}}\left\langle\left.\eta_{\mathrm{LFT}}\right|_{y} ^{-1}\right\rangle$.

That $F_{\mathrm{EXP}} \phi_{\mathrm{EXP}}=F_{\mathrm{LFT}} \phi_{\mathrm{LFT}}$ can now be shown in the following steps, explained below, proceeding from the right to the left side:

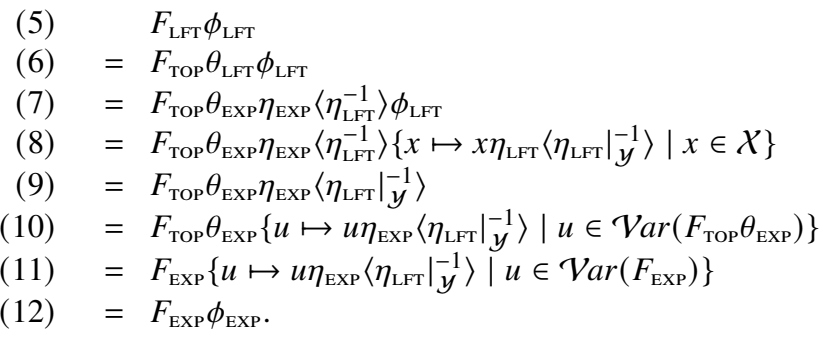

Equality of (5) to (6) follows from (3). Equality to (7) follows since by the definition of $\theta_{\mathrm{LFT}}$ and (2) it holds that $F_{\mathrm{TOP}} \theta_{\mathrm{LFT}}=F_{\mathrm{TOP}} \theta_{\mathrm{EXP}} \eta_{\mathrm{EXP}}\left\langle\eta_{\mathrm{LFT}}^{-1}\right\rangle$. Equality to (8) follows from the definition of $\phi_{\mathrm{LFT}}$ (Definition 29). Equality to (9) follows from (4), since $F_{\mathrm{TOP}} \theta_{\mathrm{EXP}} \eta_{\mathrm{EXP}}=F_{\mathrm{EXP}} \eta_{\mathrm{EXP}}=$ $F_{\mathrm{GRD}}$, where the first equality in the sequence follows from (1) and the second one holds by Definition 24.i. Equality to (10) follows with similar arguments as step (2) above: Because the range of $\eta_{\mathrm{LFT}} \mid y$ contains only $\mathcal{G}$-terms and there are no occurrences of $\mathcal{G}$-terms in $F_{\mathrm{TOP}} \theta_{\mathrm{EXP}}$, inversely applying $\eta_{\mathrm{LFT}} \mid y$ to an instance of $F_{\mathrm{TOP}} \theta_{\mathrm{EXP}}$, say $F_{\mathrm{TOP}} \theta_{\mathrm{EXP}} \xi$, yields the same result as applying to $F_{\mathrm{TOP}} \theta_{\mathrm{EXP}}$ the substitution that maps each variable $u$ occurring in $F_{\mathrm{TOP}} \theta_{\mathrm{EXP}}$ to the value of $u \xi$ after inversely applying $\eta_{\mathrm{LFT}} \mid y$. For the particular case of $\eta_{\mathrm{EXP}}$ in the role of $\xi$ this is formally stated as the equality of (9) and (10). Equality to (11) follows from (1), and equality to (12) by contracting the definition of $\phi_{\mathrm{EXP}}$, Definition 31. 
As a side remark, we note that the proof of Proposition 32 implies with its step (9) a third characterization of the formulas equated by the proposition, in terms of the ground formula $F_{\mathrm{GRD}}$ with an inversely applied substitution: $F_{\mathrm{EXP}} \phi_{\mathrm{EXP}}=F_{\mathrm{LFT}} \phi_{\mathrm{LFT}}=F_{\mathrm{GRD}}\left\langle\left.\eta_{\mathrm{LFT}}\right|_{y} ^{-1}\right\rangle$.

The following example illustrates the intermediate formulas and substitutions introduced so far to prove the interpolant lifting theorem.

Example 33 (Formulas and Substitutions Involved in the Proof of Interpolant Lifting) The diagram in Figure 10 shows instance relationships of some of the quantifier-free formulas and substitutions used to prove interpolant lifting, along with examples. A formula connected with a downward line to another formula is, after applying the substitution that labels the line, identical to the lower formula. Injective substitutions are indicated by thick lines. The shown symbolic formula and substitution names refer to an implicitly given interpolant lifting base with $\mathcal{F}=\{\mathrm{f}\}$ and $\mathcal{G}=\{\mathrm{g}\}$ and to the definitions in this section. (Exceptions are $F_{\text {ToP }}$ and $\theta_{\mathrm{EXP}}, \theta_{\mathrm{LFT}}$, which are only used locally within the proof of Proposition 32.)

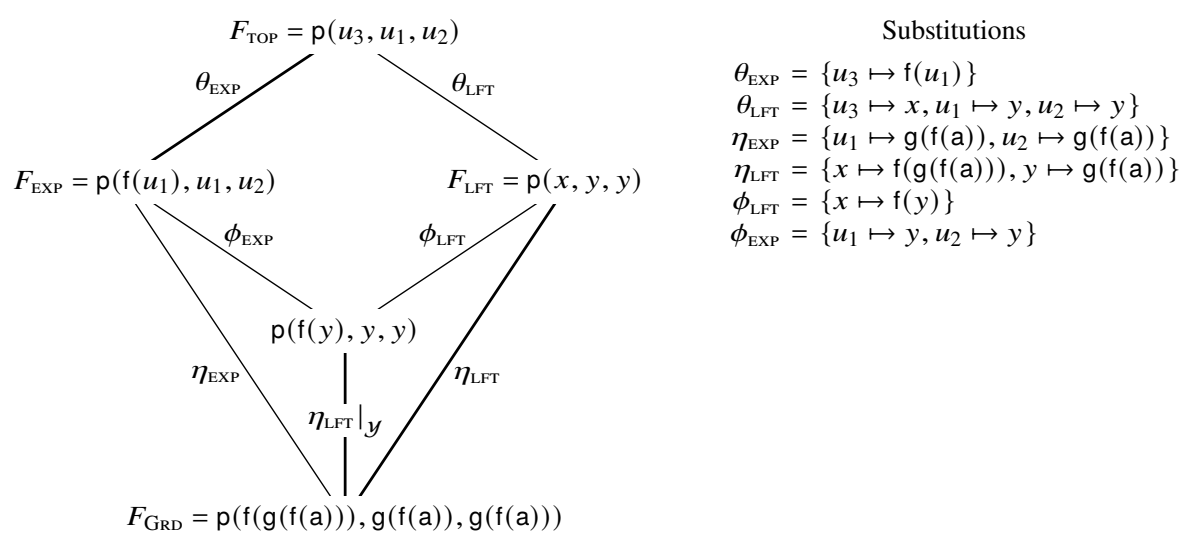

Fig. 10: Instance relationships between formulas involved in the proof of interpolant lifting with example values.

We are now ready to prove the required semantic property of the formula obtained by interpolant lifting with the following lemma.

Lemma 34 (Semantic Justification of Interpolant Lifting: From $F$ to the Interpolant)

$$
F \vDash Q_{1} v_{1} \ldots Q_{n} v_{n} H_{G R D}\left\langle\sigma^{-1}\right\rangle .
$$

Proof That the lemma holds under the assumption of the following statement (1) can be shown in the subsequent steps explained below:

$$
\begin{aligned}
& F \mid=\exists \mathcal{F} Q_{1}^{\prime} v_{1}^{\prime} \ldots Q_{m}^{\prime} v_{m}^{\prime} \forall \mathcal{Y}_{m} F_{\mathrm{LFT}} \phi_{m} \\
& \text { iff } F=Q_{1}^{\prime} v_{1}^{\prime} \ldots Q_{m}^{\prime} v_{m}^{\prime} F_{\mathrm{LFT}} \\
& \text { implies } F=Q_{1}^{\prime} v_{1}^{\prime} \ldots Q_{m}^{\prime} v_{m}^{\prime} H_{\mathrm{LFT}} \\
& \text { iff } F=Q_{1} v_{1} \ldots Q_{n} v_{n} H_{\mathrm{LFT}} \\
& \text { iff } F=Q_{1} v_{1} \ldots Q_{n} v_{n} H_{\mathrm{GRD}}\left\langle\sigma^{-1}\right\rangle \text {. }
\end{aligned}
$$

The equivalence of (1) to (2) holds since it follows from the definitions of $\phi_{i}$ and $\mathcal{Y}_{i}$ that $\phi_{m}=\varepsilon$ and $\mathcal{Y}_{m}=\emptyset$, and from the definitions of $F_{\mathrm{LFT}}$ and $\eta_{\mathrm{LFT}}$ that members of $\mathcal{F}$ do not 
occur in $F_{\mathrm{LFT}}$. The implication of (3) follows from Proposition 27.ii, equivalence to (4) from Proposition 27.iii and equivalence to (5), the statement to prove, from unfolding the definition of $H_{\mathrm{LFT}}$.

It remains to show assumption (1). We show by induction that actually for all $i \in$ $\{0, \ldots, m\}$ it holds that

$$
F \vDash \exists \mathcal{F} Q_{1}^{\prime} v_{1}^{\prime} \ldots Q_{i}^{\prime} v_{i}^{\prime} \forall \mathcal{Y}_{i} F_{\mathrm{LFT}} \phi_{i},
$$

which, of course, with the case $i=m$ includes assumption (1). We first consider the base case where $i=0$. Let $\mathcal{U} \stackrel{\text { def }}{=} \operatorname{Var}\left(F_{\mathrm{EXP}}\right)$. From the key property of $\phi_{\mathrm{EXP}}$ (Proposition 32) it follows that

(6) $\forall \mathcal{U} F_{\mathrm{EXP}} \models \forall \mathcal{Y} F_{\mathrm{EXP}} \phi_{\mathrm{EXP}}=\forall \mathcal{Y} F_{\mathrm{LFT}} \phi_{\mathrm{LFT}}$.

Condition (i) of the definition of interpolant lifting base (Definition 16) states that $F \models$ $\exists \mathcal{F} \forall \mathcal{U} F_{\mathrm{EXP}}$. With (6) this implies $F=\exists \mathcal{F} \forall \mathcal{Y} F_{\mathrm{LFT}} \phi_{\mathrm{LFT}}$, which, since $\mathcal{Y}=\mathcal{Y}_{0}$ and $\phi_{\mathrm{LFT}}=\phi_{0}$, can be written as $F \vDash \exists \mathcal{F} \forall \mathcal{Y}_{0} F_{\mathrm{LFT}} \phi_{0}$, that is, the statement to show for the base case.

We now consider the induction step. As induction hypothesis we assume that $(*)$ holds for some $i \in\{0, \ldots, m-1\}$. The induction conclusion, that is, $(*)$ with $i+1$ in place of $i$, follows from the hypothesis since for all $i \in\{0, \ldots, m-1\}$ it holds that

$$
Q_{1}^{\prime} v_{1}^{\prime} \ldots Q_{i}^{\prime} v_{i}^{\prime} \forall \mathcal{Y}_{i} F_{\mathrm{LFT}} \phi_{i} \vDash Q_{1}^{\prime} v_{1}^{\prime} \ldots Q_{i+1}^{\prime} v_{i+1}^{\prime} \forall \mathcal{Y}_{i+1} F_{\mathrm{LFT}} \phi_{i+1},
$$

which we now show. Let $i$ be a member of $\{0, \ldots, m-1\}$. The variable $v_{i+1}^{\prime}$ is then either in $\mathcal{X}$ or in $\mathcal{Y}$. We show $(* *)$ for both cases separately:

- Case $v_{i+1}^{\prime} \in \mathcal{X}$ : Then $Q_{i}^{\prime}=\exists, \phi_{i}=\left\{v_{i+1}^{\prime} \mapsto v_{i+1}^{\prime} \phi_{i}\right\} \phi_{i+1}$ and $\mathcal{Y}_{i+1}=\mathcal{Y}_{i}$. Moreover, by Proposition 30 it holds that:

(7) $v_{i+1}^{\prime} \notin \mathcal{V} \mathcal{R} n g\left(\phi_{i+1}\right)$.

(8) $\mathcal{Y}_{i+1} \cap \operatorname{Var}\left(v_{i+1}^{\prime} \phi_{i}\right)=\emptyset$.

Hence,

(9) $\quad Q_{1}^{\prime} v_{1}^{\prime} \ldots Q_{i}^{\prime} v_{i}^{\prime} \forall \mathcal{Y}_{i} F_{\mathrm{LFT}} \phi_{i}$

(10) $=Q_{1}^{\prime} v_{1}^{\prime} \ldots Q_{i}^{\prime} v_{i}^{\prime} \forall \mathcal{Y}_{i+1} F_{\mathrm{LFT}}\left\{v_{i+1}^{\prime} \mapsto v_{i+1}^{\prime} \phi_{i}\right\} \phi_{i+1}$

(11) $\quad=Q_{1}^{\prime} v_{1}^{\prime} \ldots Q_{i}^{\prime} v_{i}^{\prime} \exists v_{i+1}^{\prime} \forall \mathcal{Y}_{i+1} F_{\mathrm{LFT}} \phi_{i+1}$

(12) $=Q_{1}^{\prime} v_{1}^{\prime} \ldots Q_{i+1}^{\prime} v_{i+1}^{\prime} \forall \mathcal{Y}_{i+1} F_{\mathrm{LFT}} \phi_{i+1}$,

where the entailment of (11) by (10) is justified by (7) and (8). This concludes the proof of the induction step for the case $v_{i+1}^{\prime} \in X$.

- Case $v_{i+1}^{\prime} \in \mathcal{Y}$ : Then $Q_{i}^{\prime}=\forall, \phi_{i+1}=\phi_{i}$ and $\mathcal{Y}_{i}=\left\{v_{i+1}^{\prime}\right\} \cup \mathcal{Y}_{i+1}$. Hence

(13) $\quad Q_{1}^{\prime} v_{1}^{\prime} \ldots Q_{i}^{\prime} v_{i}^{\prime} \forall \mathcal{Y}_{i} F_{\mathrm{LFT}} \phi_{i}$

(14) $=Q_{1}^{\prime} v_{1}^{\prime} \ldots Q_{i}^{\prime} v_{i}^{\prime} \forall v_{i+1}^{\prime} \forall \mathcal{Y}_{i+1} F_{\mathrm{LFT}} \phi_{i+1}$

(15) $=Q_{1}^{\prime} v_{1}^{\prime} \ldots Q_{i+1}^{\prime} v_{i+1}^{\prime} \forall \mathcal{Y}_{i+1} F_{\mathrm{LFT}} \phi_{i+1}$,

which concludes the proof of the induction step for the case $v_{i+1}^{\prime} \in \mathcal{Y}$. 
Corollary 35 (Semantic Justification of Interpolant Lifting: From the Interpolant to $G$ )

$$
Q_{1} v_{1} \ldots Q_{n} v_{n} H_{G R D}\left\langle\sigma^{-1}\right\rangle \vDash G .
$$

Proof (Sketch) A formula $H$ is a Craig-Lyndon interpolant of formulas $F$ and $G$ if and only if $\neg H$ is a Craig-Lyndon interpolant of $\neg G$ and $\neg F$. Let $\bar{Q}_{i} \stackrel{\text { def }}{=} \forall$ if $Q_{i}=\exists$ and $\bar{Q}_{i} \stackrel{\text { def }}{=} \exists$ if $Q_{i}=\forall$. The corollary statement then can then be expressed as

$$
\neg G \models \bar{Q}_{1} v_{1} \ldots \bar{Q}_{n} v_{n} \neg H_{\mathrm{GRD}}\left\langle\sigma^{-1}\right\rangle .
$$

Given that $\left\langle F, G, \mathcal{F}, \mathcal{G}, H_{\mathrm{GRD}}, F_{\mathrm{EXP}}, \neg G_{\mathrm{EXP}}, \eta_{\mathrm{EXP}}\right\rangle$ is a lifting base, it is not difficult to verify that also

$$
\left\langle\neg G, \neg F, \mathcal{G}, \mathcal{F}, \neg H_{\mathrm{GRD}}, \neg G_{\mathrm{EXP}}, \neg F_{\mathrm{EXP}}, \eta_{\mathrm{EXP}}\right\rangle
$$

forms a lifting base. If we derive Lemma 34 from this lifting base and $\bar{Q}_{1}, \ldots, \bar{Q}_{n}$ instead of $Q_{1}, \ldots, Q_{n}$ as starting points, then the lemma reads exactly as (*).

Finally, the proof of the interpolant lifting theorem can be stated, based on Lemma 34 and considerations on syntactic properties of the formula obtained by interpolant lifting.

Proof (Theorem 21) Let $H \stackrel{\text { def }}{=} Q_{1} v_{1} \ldots Q_{n} v_{n} H_{\mathrm{GRD}}\left\langle\sigma^{-1}\right\rangle$. According to the definition of Craig-Lyndon interpolant (Definition 3 ) we have to verify semantic and syntactic properties. The semantic properties are $F \vDash H$, which has been shown as Lemma 34, and $H \vDash G$, stated as Corollary 35. The syntactic property is ${\mathcal{V} o c^{ \pm}}(H) \subseteq \mathcal{V} o c^{ \pm}(F) \cap \mathcal{V} o c^{ \pm}(G)$, or, equivalently, $\mathcal{P r e d}^{ \pm}(H) \subseteq \mathcal{P r e d}^{ \pm}(F) \cap \mathcal{P r e d}^{ \pm}(G)$ and $\mathcal{F} u n(H) \subseteq \mathcal{F} u n(F) \cap \mathcal{F u n}(G)$. The requirement on predicate occurrences hold since by conditions (vii), (ii) and (ii') of the definition of interpolant lifting base (Definition 16) it follows that

$$
\operatorname{Pred}^{ \pm}(H) \subseteq \operatorname{Pred}^{ \pm}\left(H_{\mathrm{GRD}}\right) \subseteq \operatorname{Pred}^{ \pm}\left(F_{\mathrm{EXP}}\right) \cap \operatorname{Pred}^{ \pm}\left(G_{\mathrm{EXP}}\right) \subseteq \operatorname{Pred}^{ \pm}(F) \cap \operatorname{Pred}^{ \pm}(G)
$$

The requirement on function symbols can be shown as follows: Since the construction of $H$ involves the inverse application of the substitution $\sigma$ to the ground interpolant $H_{\text {GRD }}$, which causes all terms in which members of $\mathcal{F} \cup \mathcal{G}$ occur to be replaced by variables, it holds that $\mathcal{F} u n(H) \cap(\mathcal{F} \cup \mathcal{G})=\emptyset$. From conditions (vii), (vi), (iii) and (iii') of the definition of interpolant lifting base it follows that

$\mathcal{F} u n(H) \subseteq \mathcal{F} u n\left(H_{\mathrm{GRD}}\right) \subseteq \mathcal{F} u n\left(F_{\mathrm{EXP}}\right) \cup \mathcal{F} u n\left(G_{\mathrm{EXP}}\right) \cup \mathcal{F} \cup \mathcal{G} \subseteq(\mathcal{F} u n(F) \cap \mathcal{F} u n(G)) \cup \mathcal{F} \cup \mathcal{G}$ which together with $\mathcal{F} u n(H) \cap(\mathcal{F} \cup \mathcal{G})=\emptyset$ implies $\mathcal{F} u n(H) \subseteq \mathcal{F} u n(F) \cap \mathcal{F} u n(G)$.

\section{Related Work, Refinements and Issues}

\subsection{Related Work on Interpolant Lifting}

The construction of the lifting result according to Theorem 21 has in essence been already shown by Huang [36]. A minor difference is that Huang orders variables in the quantifier prefix by the length of the associated terms, which is more constrained than the subterm relationship used in Theorem 21.

Although the construction of the lifting result can be expressed as a simple formula conversion, independently of any particular calculus, its correctness seems not trivial to prove and subtle issues arise. The proof in [36] depends with [36, Lemma 12] on an induction over 
a specific representation of a proof by resolution, paramodulation and factoring. The formula on which lifting is applied is characterized there by two properties: First, it is a relational interpolant, which means that it satisfies the constraints on a Craig interpolant except that function symbols which are not shared by both input formulas are permitted. Second, it was obtained in the first stage with a specific inductive algorithm from a propositional deduction tree that in turn was obtained by a specific method from the first-order proof. The correctness of Huang's interpolation method for first-order logic with equality seems so far not settled. In [36] it is not thoroughly proven and, as noted in [14, Sect. 6], its specification [36] leaves ambiguities concerning paramodulation. In [45, Sect. 6] it is suggested that Huang's method is in presence of equality incorrect, but the example given to substantiate this is not traceable. It may be adapted, however, to illustrate that the notion of relational interpolant alone is, at least in presence of equality, not sufficient to characterize the lifted formulas: If the objective is to compute a Craig interpolant of $F=(\mathrm{a}=\mathrm{b})$ and $G=(\mathrm{g}(\mathrm{a})=\mathrm{g}(\mathrm{b}))$, then Huang's first stage would yield the relational interpolant $a=b$, which is also Craig interpolant. It would not produce $g(a)=g(b)$, which is another relational interpolant but whose lifting, that is, $\forall v_{1} \forall v_{2} v_{1}=v_{2}$, is not a Craig interpolant of $F$ and $G$ because it is not entailed by $F$. Seemingly independently from [36], the correctness of interpolant lifting has been proven (for the case without equality) in [1, Lemma 8.2.2] based on natural deduction proofs as data structures (lifting is called abstraction in [1]). In contrast, our justification of the lifting step (for the case without equality) is based more abstractly on Herbrand's theorem instead of a resolution or natural deduction proof structure. The formula to be lifted is characterized as an actual Craig-Lyndon interpolant of two intermediate formulas whose existence is ensured but which do not have to be constructed at interpolant computation.

Huang's relational interpolants permit free variables upon which extra quantifiers will be added after lifting. As indicated in [36, p. 188], this can be done in an arbitrary way: the extra quantifiers can be existential or universal, at any position in the prefix. In our formalization, the base formulas used for lifting have to be ground. The effects described by Huang appear to be subsumed by the alternate possibilities to instantiate non-ground tableaux delivered by provers as discussed in Sect. 10.2. The method of [1] to construct the results of the first stage, called weak interpolants there, involves certain cases where quantified variables are introduced.

The special case of the lifting theorem where constants are the only functions to be replaced by variables was shown already by Craig in his proof of the interpolation theorem for equality-free relational formulas [24, Lemma 1] (as observed in [14]) and for application to an interpolation method for proofs by resolution and superposition as well as support for theory reasoning in [14]. Both proofs are independent of a particular calculus or proof data structures.

\subsection{Choices in Grounding and Side Assignment}

The CTIF method (Procedure 23) for the construction of first-order interpolants leaves at several stages alternate choices that have effect on the formula returned as interpolant. We discuss some of these here, although a thorough investigation of ways to integrate the exploration and evaluation of these into interpolant construction seems a nontrivial topic on its own.

The first considered choice concerns the tableau grounding step (step 3 of the procedure). Typically, provers instantiate variables just as much "as needed" by the calculus to compute a closed tableau. To match with our inductive interpolant extraction and interpolant lifting, 
these rigid variables in the literal labels of such free-variable tableaux have to be instantiated by ground terms. There are different possibilities to do so, all preserving the property that the tableau is a leaf-closed two-sided tableau for $F^{\prime}$ and $G^{\prime}$, but leading to different interpolants: A variable can be instantiated by a term whose functions all occur in both interpolation inputs. The term may then occur in the interpolant. Alternatively, the variable can be instantiated by a term whose outermost function symbol occurs in just one of the input formulas or has been introduced at Skolemization. By interpolant lifting the term will then be replaced with a variable whose kind, existential or universal, depends on the outermost symbol of the term, and whose quantifier position in the prefix is constrained by its subterms.

Aside of these alternate possibilities that concern the instantiation of each variable individually, there are also choices to instantiate different variables by the same term or by different terms: Arbitrary subsets of the free variables of the literal labels of the tableau can be instantiated with the same ground term, leading in the interpolant to fewer quantified variables but to more "variable sharing", that is, an increase in the number of occurrences of each variable throughout the formula.

A second choice point concerns the side assignment (step 4 of the procedure): A tableau clause can be an instance of some clause $F^{\prime}$ as well as of some clause in $G^{\prime}$, such that its side (i.e., the side labels of the nodes labeled with its literals) can be assigned to $F$ or $G$, where both assignments may lead to different interpolants. This possibility may occur if a clause in $F^{\prime}$ and a clause in $G^{\prime}$ have a common instance, including the cases where they are identical or are variants, that is, identical modulo systematic variable renaming. Effects of alternate choices at side assignment were illustrated with Example 10 on page 13.

\subsection{Preprocessing and Structure-Preserving Normalization}

Sophisticated preprocessing is a crucial component of automated reasoning systems with high performance. While formula simplifications such as removal of subsumed clauses and removal of tautological clauses preserve equivalence, others only preserve unsatisfiability. For example, purity simplification, that is, removal of clauses that contain a literal with a predicate that occurs with only one polarity in the formula. Many simplifications of the latter kind actually preserve not just unsatisfiability, but, moreover, equivalence with respect to a set of predicates, or, more precisely, a second-order equivalence

$$
\exists p_{1} \ldots \exists p_{n} F \equiv \exists p_{1} \ldots \exists p_{n} \operatorname{simplify}(F),
$$

where simplify $(F)$ stands for the result of the simplification operation applied to $F$. One might say that the semantics of the predicates not in $\left\{p_{1}, \ldots, p_{n}\right\}$ is preserved by the simplification. For the computation of Craig-Lyndon interpolants it is possible to preprocess the first as well as the negated second input formula independently from each other in ways such that the semantics of the predicates occurring in both formulas is preserved in this sense. The used preprocessors then should support parameterization with the set of these predicates (see [81, Sect. 2.5] for a discussion).

For clausal tableau methods some of these simplifications are particularly relevant as they complement tableau construction with techniques which break apart and join clauses and may thus introduce some of the benefits of resolution. Techniques for propositional logic that preserve equivalence $(*)$ for certain sets of predicates include variable elimination by resolution [29] and blocked clause elimination [40]. The preprocessing of Prover9 [57] applies by default a form of predicate elimination. In general, for first-order generalizations of such elimination-based techniques the handling of equality seems the most difficult issue. 
Predicate elimination can introduce equality also for inputs without equality. In a semantic framework where the Herbrand universe is taken as domain this can be avoided to some degree, as shown in [80] with a variant of the SCAN algorithm [32] for predicate elimination. Blocked clause elimination in first-order logic [44] comes in two variants, for formulas without and with equality, respectively.

Another way to use equivalence $(*)$ is by introducing fresh "definer" predicates for example by structure-preserving (also known as definitional) normal forms such as the Tseitin transformation and first-order generalizations of it $[71,78,28,68]$. If disjoint sets of definer predicates are used for the first and for the second interpolation input, then, by the definition of Craig-Lyndon interpolant, definer predicates do not occur in the interpolant.

In certain situations, which need further investigation, it might be useful to relax this constraint. For example, if two definer predicates have the same subformula as definiens, it is in general useful to identify both predicates, that is, to remove the defining formulas for one of them and replace its definer predicate by the other, retained, definer predicate. If the two definer predicates each stem from separate preprocessing of the first and second interpolation inputs, respectively, the merged definer predicate would occur in both inputs and might occur in the interpolant. Another example would be allowing definers occurring in the interpolant in cases where this permits a condensed representation of a formula whose equivalent without the definers would be much larger but straightforward to obtain.

\subsection{Equality Handling}

So far we considered only first-order logic without equality. Nevertheless, our method to compute interpolants can be used together with the well-known encoding of equality as a binary predicate with axioms that express its reflexivity, symmetry and transitivity as well as axioms that express substitutivity of predicates and functions. If the input formulas of interpolant computation involve equality, these axioms have to be added. The interpolation inputs are then formulas $E_{\mathrm{F}} \wedge F$ and $E_{\mathrm{G}} \rightarrow G$ instead of $F$ and $G$, respectively, where $E_{\mathrm{F}}$ and $E_{\mathrm{G}}$ are conjunctions of equality axioms: The substitutivity axioms for predicate and function symbols that occur only in one of $F$ or $G$ are placed in $E_{\mathrm{F}}$ or $E_{\mathrm{G}}$, respectively, whereas the axioms that express reflexivity, symmetry and transitivity as well as substitutivity axioms for symbols that occur in both $F$ and $G$ can be placed arbitrarily in $E_{\mathrm{F}}$, in $E_{\mathrm{G}}$, or in both of them.

For formulas without function symbols with exception of constants in which equality occurs only in one of the inputs, say $F$, more can be said about the polarity in which it can occur in the interpolant: The axioms expressing reflexivity, symmetry and transitivity can be added to $E_{\mathrm{F}}$. After adding the substitutivity axioms, for example $\forall x \forall y(\mathrm{p}(x) \wedge x=y \rightarrow \mathrm{p}(y))$, to $E_{\mathrm{F}}$ and $E_{\mathrm{G}}$ as described above, all occurrences of equality in $E_{\mathrm{G}} \rightarrow G$ are in substitutivity axioms in which they have negative polarity. Hence, in $E_{G} \rightarrow G$ they have positive polarity and in a Craig-Lyndon interpolant of $E_{\mathrm{F}} \wedge F$ and $E_{\mathrm{G}} \rightarrow G$ they must also have positive polarity. Analogously it can be shown that if equality occurs only in the other input $G$, then it can occur in the interpolant only with negative polarity. Stronger constraints on interpolants with respect to equality are stated in an interpolation theorem due to Oberschelp and Fujiwara (see [63]).

Equality handling with goal-directed clausal tableau provers is notoriously difficult. The modern leanCoP system [66] indeed encodes equality just as a predicate with axioms as mentioned, with some practical success for the price of loosing completeness due to restricted backtracking, a technique to cut off parts of the explored search space. Recently 
an equality preprocessing technique for leanCoP has been described [65], which can be understood as deriving specific clauses involving equality followed by performing simplifications. The best experimental results were obtained with an incomplete variant in which also certain non-redundant clauses are deleted and no equality axioms are added. The historic SETHEO system [61] successfully integrated a refinement of Brand's STE-modification [16], a transformation of the source axioms that makes the equality axioms redundant. A further refinement of Brand's method that takes term ordering constraints into account is described in [2]. CMProver [81,83] supports both, the axiom-based equality representation and an implementation of the transformation from [2]. In principle, variants of Brand's transformation can be expected to be applicable for interpolation, although their semantic properties shown in the literature [16,2] are just up to the preservation of satisfiability and unsatisfiability, whereas for interpolation equivalence, or at least the preservation of the second-order equivalence discussed in Sect. 10.3, is required.

\subsection{Issues with Top-Down and Bottom-Up Clausal Tableau Provers}

As demonstrated with the two different tableaux for the same inputs in Example 9 on page 11, there exist in general quite different closed clausal tableaux for a given clausal formula, leading to different extracted interpolants. For top-down methods such as model elimination [53] and the connection method [10,11], the constructed tableau is often largely determined by the chosen start clause, that is, the clause attached to the root. The addition of further clauses is then guided by the requirement that it closes an open branch through the connection condition, that is, the last literal on the branch is the complement of a literal in the clause. Such provers typically consider a specific subset of the input clauses as start clauses. Without loss of completeness the set of negative clauses can, for example, be taken as this subset, or, if a theorem is to be proven from a consistent set of axioms, the clauses representing the (negated) theorem. It remains to be investigated what choices of start clauses are particularly useful for the computation of interpolants.

Bottom-up methods such as the hypertableau calculus [3] as such typically construct variations of clausal tableaux that do not match our requirements as they may contain nonrigid variables. Translations into tableaux with only rigid variables need to be developed, which should be facilitated by the constraint that different literals in the clause of a hypertableau are not allowed to share variables. When applied to a clausal formula that is range restricted (all variables in a clause occur in a negative literal), an important case in practice, the hypertableau calculus constructs a tableau that is ground - and thus is trivially a tableau with only rigid variables that can be directly used in the CTIF procedure.

\subsection{An Implementation}

An implementation of the CTIF method, which is available as free software, is integrated in the PIE environment $[81,83]$. The construction of the tableaux for interpolation is performed there with the first-order prover CMProver that proceeds in the goal-oriented top-down way. ${ }^{14}$

\footnotetext{
14 Inspired by the Prolog Technology Theorem Prover [74] and SETHEO [51], CMProver was originally written in 1993 but had been revived in 1996 [26] and in 2016. It was evaluated in 2018 on all suitable TPTP problems, that is, problems that have a distinguished theorem, are not classified as satisfiable and are in clausal or quantified first-order form: Of these, it can solve about $76 \%$ of the 2143 problems without equality (in 9 configurations) and about $26 \%$ of the 11321 problems with equality (in 4 configurations). The timeout was 600s, the TPTP version was 7.1.0. See http://cs. christophwernhard. com/pie/cmprover/ for details.
} 
Experimental support is also provided for bottom-up tableau construction with the Hyper $[79,67,6]$ hypertableau system. The clausal tableaux used for interpolant extraction are represented as Prolog terms, providing an interface to integrate further provers.

Configurable preprocessing provides simplifications that respect preservation of predicate semantics as required for interpolation, supports structure-preserving clausification and handles the adding of equality axioms. Configurable postprocessing allows to integrate into the interpolant extraction ground formula simplifications that are aware of equality, e.g., rewrite $\mathrm{a}=\mathrm{a}$ to $\mathrm{T}$, and to apply different first-order simplifications to the overall result. Symmetric interpolation [24, Lemma 2] (the name is due to [59]) with consideration of predicate polarity is implemented as iterated Craig-Lyndon interpolation.

So far, the implemented CTIF method has not yet been experimentally compared with other implementations of interpolation in first-order logic, such as an extension of Vampire [35] and Princess [17]. Both of them are mainly targeted at applications in verification that involve theory reasoning. Experiments with applications of interpolation to ground formulas for query reformulation are described in [8]. Aside of Vampire also implementations of variations of the inductive algorithms from [36,12,58] have been tried in [8] on the basis of resolution proofs returned by the MathSAT SMT solver [22] and the E first-order prover [70]. In addition, a method based on the chase technique, implemented with $D L V$ [46] as model generator was compared. In cases where the expected reformulation is a disjunctive normal form, the $D L V$-based approach was only slightly worse than the best resolution-based approach. Another lesson reported in [8] was that the requirements on interpolation for query reformulation seem quite different than for verification. The Vampire extension, for example, seems to compute just Craig (in contrast to Craig-Lyndon) interpolants.

\section{Conclusion}

We have investigated the computation of Craig interpolants with automated theorem provers that compute clausal first-order tableaux. The presented method proceeds in two stages, similar to some interpolation methods for resolution proofs $[36,14]$. In the first stage an intermediate formula is computed by an induction on the proof representation returned by a theorem prover. The proof representation is in our case a clausal tableau. In the second stage the intermediate formula is converted to an actual interpolant by lifting, that is, replacing terms with variables and prepending a quantifier prefix.

The involved induction on clausal tableaux is an adaptation of an interpolation method for analytic tableaux that deconstructs them bottom-up [73]. The version for clausal tableaux reveals striking parallels with the induction on resolution deduction trees performed by the interpolation methods surveyed in [13]. The parallels involve some dualities and seem related to inherent correspondences of resolution and clausal tableaux. Exploring them in depth is an issue for future research. Based on a known linear simulation of tree resolution by clausal tableaux [49] we have shown that in propositional logic interpolation with clausal tableaux can linearly simulate the most prominent inductive interpolation methods for resolution proofs. The obtained clausal tableaux need to permit atomic cuts, that is, tautological clauses of the form $\neg A \vee A$. On the resolution side, tree-shaped proofs are required, such that the potential sharing of subproofs in dags seems not retainable in the simulations. While the integration of cuts into theorem proving methods that construct clausal tableaux is nontrivial [50], it is straightforward to convert a given resolution proof into a clausal tableau with atomic cuts. The simulations offer an interesting view on different interpolation methods for resolution proofs, as their differences are reflected in small variations of the clausal 
tableau translation. Future research might investigate the variations systematically, potentially discovering further methods for resolution proofs as backward translations of variations.

Our interpolation method computes Craig-Lyndon interpolants, that is, predicates occur in interpolants only in polarities in which they occur in both input formulas. In particular for applications in query reformulation also further potential properties of interpolants are of interest. For example, the Horn property or that quantifiers occur only together with atoms that relativize their variables, as for example in range restricted formulas (e.g., [33]) or in access interpolants [9]. Clausal tableaux seem to provide a suitable basis for versions of interpolation that ensure such properties. As indicated in [82], this is facilitated if they are structured in such a way that the leaves are exactly the nodes with negative literals (like hypertableaux [3], except that variables are rigid). Tableau conversions may be applied to tableaux returned by provers to achieve that form [82]. This is an issue for future research.

Interpolant lifting only creates interpolants that are in prenex form, which might be undesirable or, for forms of interpolation where all occurrences of quantifications in an interpolant must be relativized by atoms, not possible. The construction of interpolants with relativized quantifications in [9] is based on an analytic tableau method, where the introduction of quantifiers may be considered as a special form of lifting that is applied during interpolant construction to subformulas. The comparison and exploration of possible ways of lifting is another issue for future research.

That the presented approach indeed provides a basis for implementing interpolation with efficient machine-oriented theorem provers for first-order logic that can be understood as constructing clausal tableaux has been demonstrated with an implementation [81,83], which, however, has so far not been much tested or compared with other systems that are capable of computing first-order interpolants.

A major drawback compared to approaches based on resolution with paramodulation and superposition might be the inherently poor equality support of clausal tableau provers, in particular provers that proceed top down and create tableaux with rigid variables. The simulations of propositional interpolation methods for resolution proofs by clausal tableaux suggest, if equality is important, another course to practical interpolation by computing a proof with a system based on resolution and superposition, followed by translating its equality reasoning steps into applications of equality axioms and then converting the proof into a clausal tableau for interpolant extraction. Investigation of this idea, whether the conversion of a first-order proof with equality reasoning steps can also be done efficiently, and whether, in analogy to the propositional case, known interpolation methods for resolution proofs can be simulated is a further issue for future research.

\section{Acknowledgments}

This research was in part supported by Deutsche Forschungsgemeinschaft (DFG) with grant WE 5641/1-1. The author thanks anonymous reviewers of previous versions for numerous very helpful suggestions and remarks.

\section{References}

1. Baaz, M., Leitsch, A.: Methods of Cut-Elimination. Springer (2011)

2. Bachmair, L., Ganzinger, H., Voronkov, A.: Elimination of equality via transformation with ordering constraints. In: CADE-15, LNCS (LNAI), vol. 1421, pp. 175-190. Springer (1998)

3. Baumgartner, P., Furbach, U., Niemelä, I.: Hyper tableaux. In: JELIA'96, LNCS (LNAI), vol. 1126, pp. 1-17. Springer (1996) 
4. Baumgartner, P., Schmidt, R.A.: Blocking and other enhancements for bottom-up model generation methods. J. Autom. Reasoning 64, 197-251 (2020)

5. Baumgartner, P., Thorstensen, E.: Instance based methods - a brief overview. KI 24(1), 35-42 (2010)

6. Bender, M., Pelzer, B., Schon, C.: System description: E-KRHyper 1.4. In: CADE-24, LNCS (LNAI), vol. 7898, pp. 126-134. Springer (2013)

7. Benedikt, M., ten Cate, B., Tsamoura, E.: Generating low-cost plans from proofs. In: PODS'14, pp. 200-211. ACM (2014)

8. Benedikt, M., Kostylev, E.V., Mogavero, F., Tsamoura, E.: Reformulating queries: Theory and practice. In: IJCAI 2017, pp. 837-843. ijcai.org (2017)

9. Benedikt, M., Leblay, J., ten Cate, B., Tsamoura, E.: Generating Plans from Proofs: The Interpolationbased Approach to Query Reformulation. Morgan \& Claypool (2016)

10. Bibel, W.: Automated Theorem Proving. Vieweg (1982). Second edition 1987

11. Bibel, W., Otten, J.: From Schütte's formal systems to modern automated deduction. In: R. Kahle, M. Rathjen (eds.) The Legacy of Kurt Schütte, chap. 13, pp. 215-249. Springer (2020)

12. Bonacina, M.P., Johansson, M.: On interpolation in decision procedures. In: TABLEAUX 2011, LNCS (LNAI), vol. 6793, pp. 1-16. Springer (2012)

13. Bonacina, M.P., Johansson, M.: Interpolation systems for ground proofs in automated deduction: a survey. J. Autom. Reasoning 54(4), 353-390 (2015)

14. Bonacina, M.P., Johansson, M.: On interpolation in automated theorem proving. J. Autom. Reasoning 54(1), 69-97 (2015)

15. Borgida, A., de Bruijn, J., Franconi, E., Seylan, I., Straccia, U., Toman, D., Weddell, G.: On finding query rewritings under expressive constraints. In: SEBD 2010. Esculapio Editore (2010)

16. Brand, D.: Proving theorems with the modification method. SIAM J. Computing 4(4), 412-430 (1975)

17. Brillout, A., Kroening, D., Rümmer, P., Wahl, T.: An interpolating sequent calculus for quantifier-free Presburger arithmetic. J. Autom. Reasoning 47(4), 341-367 (2011)

18. Bry, F., Manthey, R.: SATCHMO: A theorem prover implemented in Prolog. In: CADE-9, LNCS, vol. 310, pp. 415-434. Springer (1988)

19. Bárány, V., Benedikt, M., ten Cate, B.: Rewriting guarded negation queries. In: MFCS 2013, LNCS, vol. 8087, pp. 98-110. Springer (2013)

20. Chang, C.L., Lee, R.C.T.: Symbolic Logic and Automated Theorem Proving. Academic Press (1973)

21. Christ, J., Hoenicke, J.: Instantiation-based interpolation for quantified formulae. In: Decision Procedures in Software, Hardware and Bioware, vol. 10161. Schloss Dagstuhl - Leibniz-Zentrum für Informatik (2010)

22. Cimatti, A., Griggio, A., Schaafsma, B.J., Sebastiani, R.: The MathSAT5 SMT solver. In: TACAS 2013, LNCS, vol. 7795, pp. 93-107. Springer (2013)

23. Craig, W.: Linear reasoning. A new form of the Herbrand-Gentzen theorem. J. Symb. Log. 22(3), 250-268 (1957)

24. Craig, W.: Three uses of the Herbrand-Gentzen theorem in relating model theory and proof theory. J. Symb. Log. 22(3), 269-285 (1957)

25. Craig, W.: The road to two theorems of logic. Synthese 164(3), 333-339 (2008)

26. Dahn, I., Wernhard, C.: First order proof problems extracted from an article in the Mizar mathematical library. In: FTP'97, RISC-Linz Report Series No. 97-50, pp. 58-62. Joh. Kepler Univ., Linz, Austria (1997)

27. Dershowitz, N., Jouannaud, J.: Notations for rewriting. Bull. EATCS 43, 162-174 (1991)

28. Eder, E.: An implementation of a theorem prover based on the connection method. In: AIMSA'84, pp. 121-128. North-Holland (1985)

29. Eén, N., Biere, A.: Effective preprocessing in SAT through variable and clause elimination. In: SAT '05, LNCS, vol. 3569, pp. 61-75. Springer (2005)

30. Fagin, R., Kolaitis, P.G., Miller, R.J., Popa, L.: Data exchange: semantics and query answering. Theor. Comput. Sci. 336(1), 89-124 (2005)

31. Fitting, M.: First-Order Logic and Automated Theorem Proving, 2nd edn. Springer (1995)

32. Gabbay, D., Ohlbach, H.J.: Quantifier elimination in second-order predicate logic. In: KR'92, pp. 425435. Morgan Kaufmann (1992)

33. van Gelder, A., Topor, R.W.: Safety and translation of relational calculus queries. ACM Transactions on Database Systems 16(2), 235-278 (1991)

34. Grau, B.C., Horrocks, I., Krötzsch, M., Kupke, C., Magka, D., Motik, B., Wang, Z.: Acyclicity notions for existential rules and their application to query answering in ontologies. JAIR 47, 741-808 (2013)

35. Hoder, K., Holzer, A., Kovács, L., Voronkov, A.: Vinter: A Vampire-based tool for interpolation. In: APLAS 2012, LNCS, vol. 7705, pp. 148-156. Springer (2012)

36. Huang, G.: Constructing Craig interpolation formulas. In: COCOON '95, LNCS, vol. 959, pp. 181-190. Springer (1995) 
37. Hudek, A., Toman, D., Wedell, G.: On enumerating query plans using analytic tableau. In: TABLEAUX 2015, LNCS (LNAI), vol. 9323, pp. 339-354. Springer (2015)

38. Hähnle, R.: Tableaux and related methods. In: A. Robinson, A. Voronkov (eds.) Handb. of Autom. Reasoning, vol. 1, chap. 3, pp. 101-178. Elsevier (2001)

39. Jacobs, S., Waldmann, U.: Comparing instance generation methods for automated reasoning. J. Autom. Reasoning 38(1-3), 57-78 (2007)

40. Järvisalo, M., Biere, A., Heule, M.: Blocked clause elimination. In: TACAS 2010, LNCS, vol. 6015, pp. 129-144 (2010)

41. Kaliszyk, C.: Efficient low-level connection tableaux. In: TABLEAUX 2015, LNCS (LNAI), vol. 9323, pp. 102-111. Springer (2015)

42. Kaliszyk, C., Urban, J.: FEMaLeCoP: Fairly efficient machine learning connection prover. In: LPAR-20, LNCS, vol. 9450, pp. 88-96. Springer (2015)

43. Kiesl, B., Suda, M.: A unifying principle for clause elimination in first-order logic. In: CADE 26, LNCS (LNAI), vol. 10395, pp. 274-290. Springer (2017)

44. Kiesl, B., Suda, M., Seidl, M., Tompits, H., Biere, A.: Blocked clauses in first-order logic. In: LPAR-21, EPiC, vol. 46, pp. 31-48 (2017)

45. Kovács, L., Voronkov, A.: First-order interpolation and interpolating proof systems. In: LPAR-21, EPiC, vol. 46, pp. 49-64. EasyChair (2017)

46. Leone, N., Pfeifer, G., Faber, W., Eiter, T., Gottlob, G., Perri, S., Scarcello, F.: The DLV system for knowledge representation and reasoning. ACM Trans. Comput. Log. 7(3), 499-562 (2006)

47. Letz, R.: Clausal tableaux. In: W. Bibel, P.H. Schmitt (eds.) Automated Deduction - A Basis for Applications, vol. I, pp. 43-72. Kluwer Academic Publishers (1998)

48. Letz, R.: First-order tableau methods. In: R.H. Marcello D’Agostino Dov M. Gabbay, J. Posegga (eds.) Handb. of Tableau Methods, pp. 125-196. Kluwer Academic Publishers (1999)

49. Letz, R.: Tableau and connection calculi. structure, complexity, implementation. Habilitationsschrift, TU München (1999). Available from http://www2.tcs.ifi.lmu.de/ letz/habil.ps, accessed 7 June 2020

50. Letz, R., Mayr, K., Goller, C.: Controlled integration of the cut rule into connection tableaux calculi. J. Autom. Reasoning 13(3), 297-337 (1994)

51. Letz, R., Schumann, J., Bayerl, S., Bibel, W.: SETHEO: A high-performance theorem prover. J. Autom. Reasoning 8(2), 183-212 (1992)

52. Letz, R., Stenz, G.: Model elimination and connection tableau procedures. In: A. Robinson, A. Voronkov (eds.) Handb. of Autom. Reasoning, vol. 1, pp. 2015-2114. Elsevier (2001)

53. Loveland, D.W.: Automated Theorem Proving: A Logical Basis. North-Holland, Amsterdam (1978)

54. Lyndon, R.: An interpolation theorem in the predicate calculus. Pacific Journal of Mathematics 9, 129-142 (1959)

55. Maier, D., Mendelzon, A.O., Sagiv, Y.: Testing implications of data dependencies. ACM Trans. Database Syst. 4(4), 455-469 (1979)

56. Marx, M.: Queries determined by views: Pack your views. In: PODS '07, pp. 23-30. ACM (2007)

57. McCune, W.: Prover9 and mace4 (2005-2010). http: //www.cs . unm. edu/ mccune/prover9

58. McMillan, K.L.: Interpolation and SAT-based model checking. In: CAV 2003, LNCS, vol. 2725, pp. 1-13. Springer (2003)

59. McMillan, K.L.: Applications of Craig interpolants in model checking. In: TACAS 2005, LNCS, vol. 3440, pp. 1-12. Springer (2005)

60. McMillan, K.L.: An interpolating theorem prover. Theor. Comput. Sci. 345(1), 101-121 (2005)

61. Moser, M., Ibens, O., Letz, R., Steinbach, J., Goller, C., Schumann, J., Mayr, K.: SETHEO and E-SETHEO - the CADE-13 systems. J. Autom. Reasoning 18(2), 237-246 (1997)

62. Motik, B., Shearer, R., Horrocks, I.: Hypertableau reasoning for description logics. JAIR 36, 165-228 (2009)

63. Motohashi, N.: Equality and Lyndon's interpolation theorem. J. Symb. Log. 49(1), 123-128 (1984)

64. Nash, A., Segoufin, L., Vianu, V.: Views and queries: Determinacy and rewriting. ACM Trans. Database Systems 35(3) (2010)

65. Oliver, B.E., Otten, J.: Equality preprocessing in connection calculi. In: PAAR-2020, CEUR Workshop Proc. CEUR-WS.org (2020)

66. Otten, J.: Restricting backtracking in connection calculi. AI Communications 23(2-3), 159-182 (2010)

67. Pelzer, B., Wernhard, C.: System description: E-KRHyper. In: CADE-21, LNCS (LNAI), vol. 4603, pp. 503-513. Springer (2007)

68. Plaisted, D.A., Greenbaum, S.: A structure-preserving clause form translation. J. Symb. Comput. 2, 293-304 (1986)

69. Reckhow, R.A.: On the lengths of proofs in the propositional calculus. Ph.D. thesis, University of Toronto (1975). Available from https://www.cs.toronto.edu/ sacook/homepage/reckhow_thesis. pdf, accessed 7 June 2020 
70. Schulz, S., Cruanes, S., Vukmirovic, P.: Faster, higher, stronger: E 2.3. In: CADE 27, LNCS, vol. 11716, pp. 495-507. Springer (2019)

71. Scott, D.: A decision method for validity of sentences in two variables. J. Symb. Log. 27(4), 477 (1962)

72. Segoufin, L., Vianu, V.: Views and queries: determinacy and rewriting. In: PODS 2005, pp. 49-60. ACM (2005)

73. Smullyan, R.M.: First-Order Logic. Springer (1968). Also republished with corrections by Dover publications, 1995

74. Stickel, M.E.: A Prolog technology theorem prover: implementation by an extended Prolog compiler. J. Autom. Reasoning 4(4), 353-380 (1988)

75. Tarski, A.: Einige methologische Untersuchungen zur Definierbarkeit der Begriffe. Erkenntnis 5, 80-100 (1935)

76. Toman, D., Weddell, G.: Fundamentals of Physical Design and Query Compilation. Morgan \& Claypool (2011)

77. Toman, D., Weddell, G.: An interpolation-based compiler and optimizer for relational queries (system design report). In: IWIL 2017 Workshop and LPAR-21 Short Presentations, Kalpa, vol. 1. EasyChair (2017)

78. Tseitin, G.S.: On the complexity of derivation in propositional calculus. In: A.O. Slisenko (ed.) Studies in Constructive Mathematics and Mathematical Logic, vol. Part II, pp. 115-125. Steklov Mathematical Institute (1970)

79. Wernhard, C.: System Description: KRHyper. Tech. Rep. Fachberichte Informatik 14-2003, Universität Koblenz-Landau, Koblenz, Germany (2003)

80. Wernhard, C.: Semantic knowledge partitioning. In: JELIA 04, LNAI, vol. 3229, pp. 552-564. Springer (2004)

81. Wernhard, C.: The PIE system for proving, interpolating and eliminating. In: PAAR-2016, CEUR Workshop Proc., vol. 1635, pp. 125-138. CEUR-WS.org (2016)

82. Wernhard, C.: Craig interpolation and access interpolation with clausal first-order tableaux. CoRR abs/1802.04982 (2018). (Tech. rep. Technische Universität Dresden, KRR 18-01)

83. Wernhard, C.: Facets of the PIE environment for proving, interpolating and eliminating on the basis of first-order logic. In: DECLARE 2019, LNCS (LNAI), vol. 12057, pp. 160-177 (2020) 